Annales Geophysicae (2003) 21: 1753-1771 C European Geosciences Union 2003

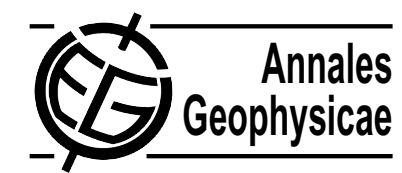

\title{
Modeling transverse heating and outflow of ionospheric ions from the dayside cusp/cleft. 1 A parametric study
}

\author{
M. Bouhram ${ }^{1, *}$, M. Malingre ${ }^{1}$, J. R. Jasperse ${ }^{2}$, and N. Dubouloz ${ }^{3}$ \\ ${ }^{1}$ CETP-CNRS, 4 Avenue de Neptune, 94107 Saint-Maur Cedex, France \\ ${ }^{2}$ Air Force research laboratory, Hanscom AFB, MA 01731-3010, USA \\ ${ }^{3}$ LPCE-CNRS, 45071 Orleans Cedex 02, France \\ *now at: Max-Planck-Institut für Extraterrestrische Physik, 85741 Garching, Germany
}

Received: 15 October 2002 - Revised: 11 February 2003 - Accepted: 27 February 2003

\begin{abstract}
The transport patterns of non-thermal $\mathrm{H}^{+}$and $\mathrm{O}^{+}$field-aligned flows from the dayside cusp/cleft, associated with transverse heating by means of wave-particle interactions and in combination with the poleward motion due to the magnetospheric convection are investigated. This has been accomplished by developing a steady-state, twodimensional, trajectory-based code. The ion heating is modelled by means of a Monte Carlo technique, via the process of ion cyclotron resonance (ICR), with the electromagnetic left-hand circular polarized component of a broad-band, extremely low-frequency (BBELF) turbulence. The altitude dependence of ICR heating from $1000 \mathrm{~km}$ to 3 Earth radii $\left(R_{E}\right)$ is modelled by a power law spectrum, with an index $\alpha$, and a parameter $w_{0}$ that is proportional to the spectral density at a referenced gyrofrequency. Because of the finite latitudinal extent of the cusp/cleft, the incorporation of the horizontal convection drift leads to a maximum residence time $t_{D}$ of the ions when being energized. A large set of simulations has been computed so as to study the transport patterns of the $\mathrm{H}^{+}$and $\mathrm{O}^{+}$bulk parameters as a function of $t_{D}, \alpha$, and $w_{0}$. Residence time effects are significant in $\mathrm{O}^{+}$density patterns while negligible for $\mathrm{H}^{+}$. When comparing the results with analytical one-dimensional theories (Chang et al., 1986; Crew et al., 1990), we find that mean ion energies and pitch angles at the poleward edge of the heating region are slightly influenced by $t_{D}$ and may be used as a probe of ICR parameters $\left(\alpha, w_{0}\right)$. Conversely, poleward of the heating region, upward velocity and mean energy dispersive patterns depend mainly on $t_{D}$ (e.g. the magnitude of the convection drift) with latitudinal profiles varying versus $t_{D}$. In short, the main conclusion of the paper is that any triplet $\left(t_{D}, \alpha, w_{0}\right)$ leads to a unique transport-pattern feature of ion flows associated with a cusp/cleft ionospheric source. In a companion paper, by using high-altitude $\left(1.5-3 R_{E}\right)$ ion observations as constraints, the results from the parametric study are used to determine the altitude dependence of transverse ion heating
\end{abstract}

Correspondence to: M. Bouhram (bouhram@mpe.mpg.de) during a significant number of passes of the Interball-2 satellite.

Key words. Magnetospheric physics (auroral phenomena) Space plasma physics (numerical simultation studies; waveparticle interactions)

\section{Introduction}

The energization and outflow of ionospheric ions at auroral latitudes have been an area of active research over the past three decades, since it was first observed by Shelley et al. (1972). Many observations and theories pointed out that the ion outflow might be of sufficient strength to supply the outer regions of the magnetosphere (see the reviews by André and Yau, 1997; and Moore et al., 1999). The enlarged cusp/cleft region has been identified as a major source of ionospheric ions for the magnetosphere (Lockwood et al., 1985a; Thelin et al., 1990). This region is located in the dayside auroral zone, extending between about 9 and $15 \mathrm{~h}$ in magnetic local time (MLT) with a latitudinal width of a few degrees.

In the cusp/cleft, much of the ionospheric ion outflow is directly caused by energization of the major ion species $\left(\mathrm{H}^{+}\right.$, $\mathrm{O}^{+}$) transverse to the geomagnetic field (André and Yau, 1997, Øieroset et al., 2000). In these regions, the heated distributions form so-called conics in velocity space, with a peak flux in an oblique direction to the geomagnetic field. Statistical studies of the evolution of ion conics versus altitude in the cusp/cleft, from 1 to 4 Earth radii $\left(R_{E}\right)$, using DE-1 (Peterson et al., 1992) and up to $10000 \mathrm{~km}$, using Akebono data (Miyake et al., 1993), pointed out that their characteristic energies increase while their apex angle (the cone angle with respect to the upward direction) decreases much more slowly than expected from adiabatic motion. Both of these findings are consistent with gradual heating of ion conics as they move upward along the geomagnetic field lines. 
Depending on the altitude of observations, typical temperatures of ion conics range from $10 \mathrm{eV}$ up to at least a few $\mathrm{keV}$ (Moore et al., 1999).

In the dayside polar cap poleward of the cusp/cleft, observations by DE- 1 revealed that outflowing ions, first heated in the cusp/cleft, overcome gravity via the mirror force and drift poleward under the effect of the magnetospheric convection to form the so-called ion fountain (Lockwood et al., 1985b). A scheme of a satellite moving across the ion fountain is given in Fig. 1. When observed at high altitudes, outflowing ion distributions are conical at high energies in the heating region, and field-aligned at lower energies poleward to the heating region (Horwitz, 1986; Knudsen et al., 1994; Dubouloz et al., 1998). Qualitatively, this picture can be formed by ion transverse heating in a region of finite latitudinal extent, followed by adiabatic convective flow to the satellite orbit. Poleward of the heating region, the latter stage contributes to a velocity-filter effect as evidenced by the latitude-energy dispersion on ion data. Many models based on ion trajectory calculations have been developed in order to demonstrate that the high-altitude observed outflows originate from the cusp/cleft (Horwitz and Lockwood, 1985; Knudsen et al., 1994; Dubouloz et al., 1998, 2001). However, the effect of non-adiabatic processes, such as transverse heating, have not been incorporated when modeling the ion transport in the cusp/cleft.

Because all ionospheric species achieved non-thermal energies when observed in the cusp/cleft, the heating is caused by the essentially perpendicular component of electric fields of oscillating within some frequency range (André and Yau, 1997). Recent statistical observations from the Freja (Norqvist et al., 1998) and FAST (Lund et al., 2000) satellites pointed out that a major part of ion transverse heating events are associated with an enhancement of the broadband, extremely low-frequency (BBELF) wave turbulence. The BBELF turbulence covers frequencies from less than $1 \mathrm{~Hz}$ up to several hundred $\mathrm{Hz}$, thus including the $\mathrm{H}^{+}$and $\mathrm{O}^{+}$gyrofrequencies at altitudes from about $1000 \mathrm{~km}$ up to a few $R_{E}$. Its profile versus frequency exhibits generally a power law spectrum (Kintner, 1976; Gurnett et al., 1984), which may be modelled by an index $\alpha$. In terms of ion energization, the theory of ion cyclotron resonance (ICR) heating by the electromagnetic left-hand circular polarized component around the ion gyrofrequencies (Chang et al., 1986) seems the most plausible mechanism. Adopting a power law altitude-dependent ion heating profile and computing MonteCarlo simulations, many authors confirmed that only a small fraction of the wave intensity in the BBELF spectrum is needed to cause the observed ion energies through ICR heating (Retterer et al., 1987; Crew et al., 1990; André et al., 1990; Norqvist et al., 1996). Except for the work of André et al. (1990), where detailed wave and particle observations available for one event have been used to carry out a 2-D Monte Carlo simulation, all the previous models neglected the horizontal convection drift. Because of the finite latitudinal extent of the cusp/cleft, the effect of a poleward convection leads to a limited residence time $t_{D}$ of ions when being
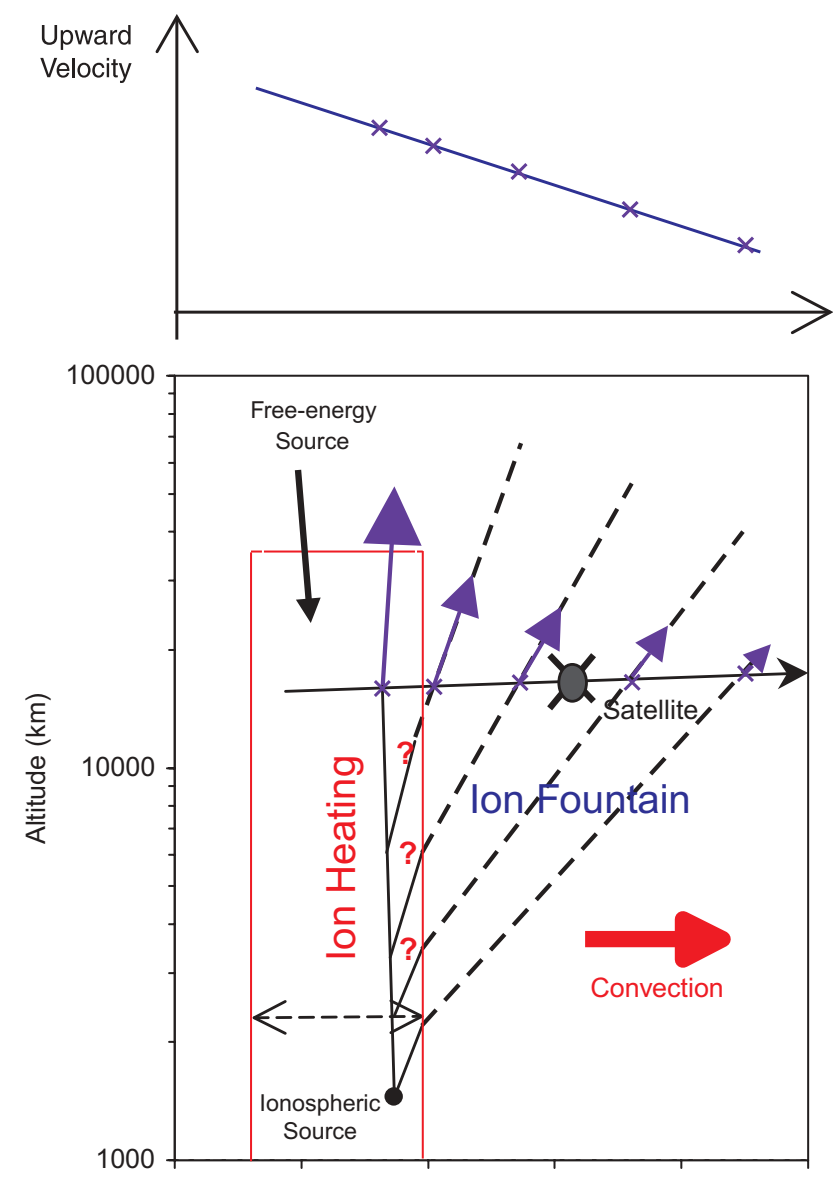

Invariant Latitude

Fig. 1. Sketch illustrating the transport patterns of ion outflows from the cusp/cleft in conjunction with a satellite crossing. The trajectories are projected in a meridian plane and are functions of invariant latitude and altitude.

heated.

In the present study, a steady-state, 2-D, Monte Carlo model has been developed. A large set of simulations is computed so as to study the effects of different geophysical parameters, such as the altitude dependence of the ion heating rate or the magnetospheric convection drift, on the transport patterns of $\mathrm{H}^{+}$and $\mathrm{O}^{+}$ions. The aim of this parametric study is focussed on determining the altitude dependence of ion transverse heating for a specific event, providing that highaltitude (below $3 R_{E}$ ) ion observations inside and poleward of the cusp/cleft are available.

The outline of the paper is as follows. A description of the simulation model is given in Sect. 2, along with a summary of the key parameters used as input to a simulation. For a few simulations, brief results and some tests are discussed in Sect. 3. The results of the parametric study applied to $\mathrm{H}^{+}$ and $\mathrm{O}^{+}$ion flows are discussed quantitatively in Sect. 4. A summary of conclusions is presented in Sect. 5 . 

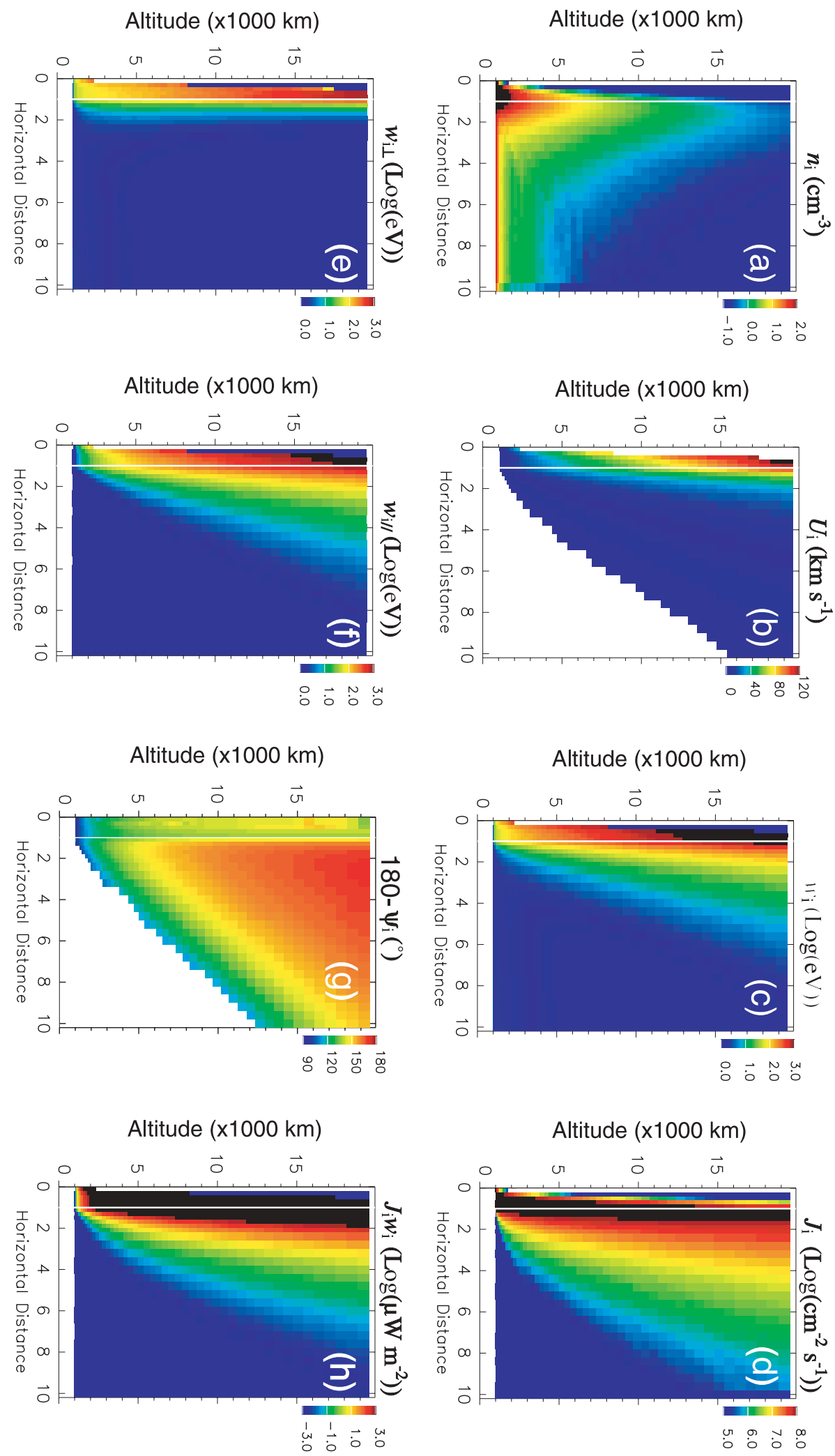

Fig. 2. Transport patterns of $\mathrm{O}^{+}$moments in the $(X, s)$ plane : (a) density in $\mathrm{cm}^{-3}$ for a value of $5000 \mathrm{~cm}^{-3}$ at the low-altitude boundary, (b) upward mean velocity in $\mathrm{km} \mathrm{s}^{-1}$, (c) mean energy in $\mathrm{eV}$, (d) fluence in $\log \left(\mathrm{cm}^{-2} \mathrm{~s}^{-1}\right)$, (e) perpendicular mean energy in eV, (f) parallel mean energy in $\mathrm{eV},(\mathbf{g})$ mean pitch angle in ${ }^{\circ}$, and (h) energy density flux in $\log \left(\mu \mathrm{W} \mathrm{m}^{-2}\right)$. The vertical white line corresponds to the poleward heating boundary $(X=1)$. 


\section{Model}

\subsection{Model geometry and applicability}

The model geometry considered here is similar to the one used by André et al. (1990). According to the scheme in Fig. 1, the coordinate system is defined by two spatial dimensions: $s$ and $X$, where $s$ denotes the length of the geomagnetic field line from its footprint, plus the Earth's radius. For high-latitude regions associated with opened fieldlines and in our altitude range $\left(s<4 R_{E}\right)$, the curvature of the magnetic field lines may be neglected and $\mathrm{s}$ is close to the geocentric distance. On the other hand, we take into consideration the divergence of the magnetic field lines by introducing an altitude dependence proportional to $1 / s^{3}$. The dayside heating regions, such as the cusp or the cleft, have longitudinal boundaries at equal latitudes (Newell and Meng, 1992). Hence, by applying a 2-D model to a data set, it is assumed that the heating region is longitudinally homogeneous. The poleward drift $\boldsymbol{v}_{p}$ caused by the $\boldsymbol{E} \times \boldsymbol{B}$ convection is taken into consideration by introducing a poleward abscissa $x$, where $x$ is a measurement of the poleward distance from the equatorward border of the heating region. For convenience, we introduce a distance $X$ normalized with respect to the thickness of the region. The relation between $X$ and the invariant latitude $\Lambda$ is given by:

$X=\left(\Lambda-\Lambda_{e q}\right) / \Delta$,

where $\Lambda_{e q}$ is the invariant latitude of the equatorial boundary and $\Delta$ the latitudinal width of the heating region.

In the present study, in order to have a reasonable number of free parameters in the simulation, we consider a constant drift $\boldsymbol{v}_{p}$ along the $X$ axis. In the $(X, s)$ frame, the effect of the drift leads to a limited residence time for ions given by $t_{D}=\Delta / v_{p}$, where $\Delta$ is the width of the heating region in kilometers. In the high-latitude ionosphere, $v_{p}$ ranges from 100 to $1000 \mathrm{~m} \mathrm{~s}^{-1}$, and $\Delta$ is about a hundred kilometers. Therefore, $t_{D}$ varies typically between 100 and $1000 \mathrm{~s}$.

Ionospheric convection patterns depend strongly on the interplanetary magnetic field (IMF) orientation (Ruohoniemi and Greenwald, 1996). In the same way, the position of the cusp may be strongly modulated by the IMF orientation (Chen et al., 1997). Hence, when assuming a constant drift in the model, it implies steady IMF conditions during the transport of the ion field-aligned flows between the ionosphere and the satellite orbit.

The simulation region extends in $\mathrm{s}$ from the topside ionosphere (at $1000 \mathrm{~km}$ altitude) to $4 R_{E}$, and in latitude from $X=0$ to $X=10$. As onset to a simulation, $\mathrm{H}^{+}$and $\mathrm{O}^{+}$test particles are launched from the topside auroral ionosphere ( $s=s_{0}$ and $\left.0<X<1\right)$ and possibly along the equatorward edge of the heating region. When considering homogeneous heating region in latitude and a constant drift, the ions associated with those two flux tubes do not mix. Hence, the transport of those populations may be computed as independent elements, with the equatorward population leading to a more accurate description of the low-latitude side of the heating region.

The structure of the steady-state ion flow patterns is carried out from the computed ion trajectories. The ions dynamics include the effect of the wave-particle interactions (WPI), the different macroscopic forces (gravity $\boldsymbol{g}$, mirror force), and the poleward drift motion caused by the convection. On the other hand, the model neglects the curvature of the field lines and therefore their effects: curvature and gradient drifts, and centrifugal acceleration (Cladis, 1986). The effects of WPI are modelled using a Monte Carlo technique, as described in the next section.

\subsection{Ion heating model}

\subsubsection{Kinetic formulation}

For a type of ion $i$, the time evolution of its distribution function $F_{i}(\boldsymbol{r}, \boldsymbol{V}, t)$ in the phase space $(\boldsymbol{r}, \boldsymbol{V})$ is given by the kinetic equation (Ichimaru, 1973):

$$
\begin{aligned}
& \left\{\frac{\partial}{\partial t}+\boldsymbol{V} \cdot \frac{\partial}{\partial \boldsymbol{r}}+\left[\boldsymbol{g}+\frac{q_{i}}{m_{i}}(\boldsymbol{E}+\boldsymbol{V} \times \boldsymbol{B})\right] \cdot \frac{\partial}{\partial \boldsymbol{V}}\right\} \\
& F_{i}(\boldsymbol{r}, \boldsymbol{V})=\frac{\partial}{\partial \boldsymbol{V}} \cdot \boldsymbol{D}_{i} \cdot \frac{\partial}{\partial \boldsymbol{V}} F_{i}(\boldsymbol{r}, \boldsymbol{V}),
\end{aligned}
$$

where $q_{i}$ and $m_{i}$ are the ion charge and mass, respectively. The right-hand side term of Eq. (2) expresses the heating of ions resulting from the wave-particle interactions, as described by a quasi-linear velocity diffusion tensor $\boldsymbol{V}_{i} \boldsymbol{D}_{i}$. Because $F_{i}(\boldsymbol{r}, \boldsymbol{V}, t)$ is gyrotropic in our problem, and its measurement takes place on times greater than the ion cyclotron period, we may consider the evolution of the distribution $f_{i}$, which is the gyrophase average of $F_{i}(\boldsymbol{r}, \boldsymbol{V})$. The phase space velocity is then decomposed in two components $V_{\|}$ and $V_{\perp}$, parallel and perpendicular to $\boldsymbol{B}$, respectively. Considering the approximations discussed above (i.e. neglecting the field-line curvature, including the convection drift only), the evolution of $f_{i}\left(s, X, V_{\|}, V_{\perp}\right)$ is given by the equation $\boldsymbol{L} f_{i}\left(s, X, V_{\|}, V_{\perp}\right)=0$ (Crew and Chang, 1985), where $\boldsymbol{L}$ is a kinetic operator, that includes the phase space flow, and can be expressed as:

$$
\begin{aligned}
& \boldsymbol{L}=V_{\|} \frac{\partial}{\partial s}+\frac{1}{t_{D}} \frac{\partial}{\partial X}+\frac{q_{i}}{m_{i}} \frac{d \phi}{d s} \frac{\partial}{\partial V_{\|}}-\frac{1}{2} \frac{d(\ln B)}{d s} \\
& \left(V_{\perp}^{2} \frac{\partial}{\partial V_{\|}}-V_{\|} V_{\perp} \frac{\partial}{\partial V_{\perp}}\right)-\frac{1}{V_{\perp}} \frac{\partial}{\partial V_{\perp}}\left(V_{\perp} D_{i \perp} \frac{\partial}{\partial V_{\perp}}\right) .
\end{aligned}
$$

The spatial variations of $f_{i}$ are entirely controlled by the first two terms in the right-hand side. The first term describes the motion along the magnetic field lines, while the second term $\left(\left(1 / t_{D}\right) \partial / \partial X \equiv v_{p} \partial / \partial x\right)$ follows the poleward motion due to the $\boldsymbol{E} \times \boldsymbol{B}$ convection. The next terms force the distribution to respond to the action of macroscopic forces, such as the potential gradient $d \phi / d s=-\left(E_{\|}+\left(m_{i} / q_{i}\right) g(s)\right)$ due to the gravity $g(s)$ and a possible parallel electric field $E_{\|}$(third term) and the mirror conversion (fourth term). We remind the 



Fig. 3. Transport patterns of $\mathrm{H}^{+}$moments in the $(X, s)$ plane. The format is the same as in Fig. 2. 

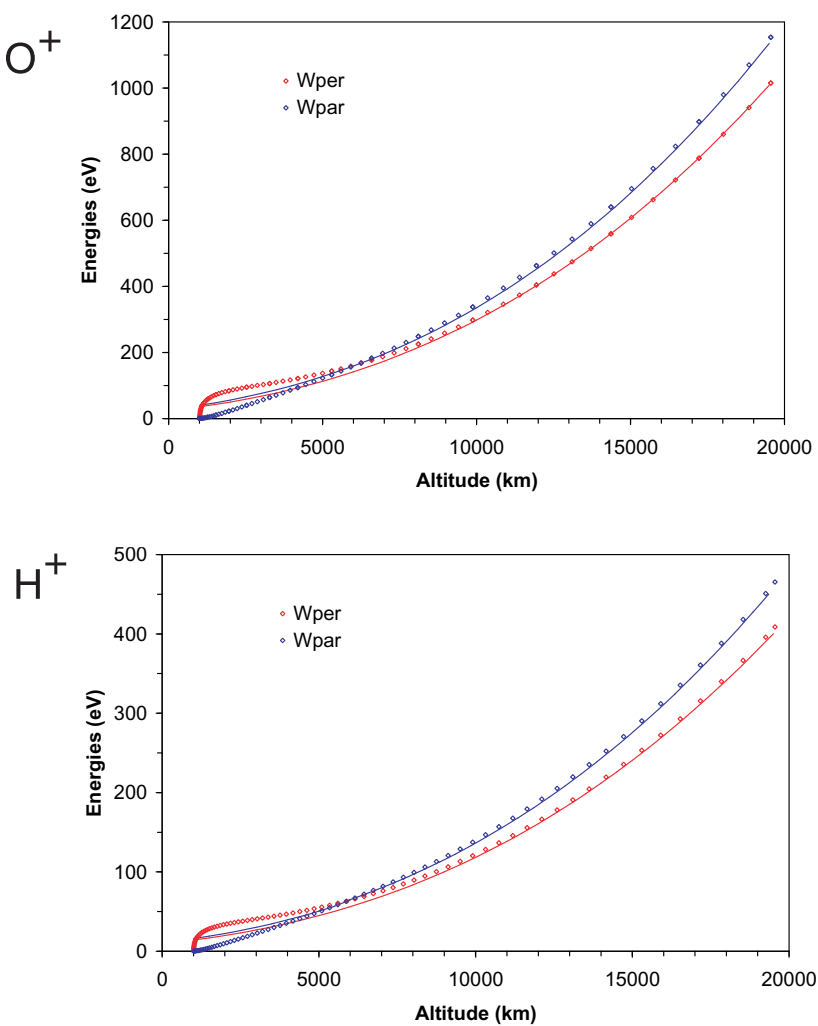

Fig. 4. Evolution of the perpendicular (red points) and parallel (blue points) energies of $\mathrm{O}^{+}$(top) and $\mathrm{H}^{+}$(bottom) test particles inside the heating region as a function of altitude. The corresponding solid curves are inferred from the mean ion theory of Chang et al. (1986).

reader that the mirror term has this simple, one-dimensional form because of the neglect of curvature and gradient drifts and centrifugal acceleration, as discussed in Sect. 1. In the case of ICR heating at the ion gyrofrequency $f_{c i}$, the component of the diffusion tensor perpendicular to $\boldsymbol{B}$ is dominant, i.e. $D_{i \perp} \gg D_{(i, \|)}$. For wavelengths greater than the ion gyroradius, $D_{i \perp}$ can be expressed as (Chang et al., 1986; Crew et al., 1990):

$D_{i, \perp}(s) \approx \frac{q_{i}^{2}}{4 m_{i}^{2}} S_{L}\left[f_{c i}(s)\right]$

where $S_{L}\left[f_{c i}\right]$ is the left-hand component of the electric field spectral density at $f_{c i}$. This results in a useful simplification of having a velocity-independent diffusion term.

\subsubsection{A Monte Carlo technique}

The solution $f_{i}\left(s, X, V_{\|}, V_{\perp}\right)$ of Eq. (3) is carried out using a Monte Carlo technique, as usually applied to investigate this kind of problem (Retterer et al., 1983, 1989). In the highaltitude cusp/cleft region, the ionospheric population is a minority constituent of the plasma (when compared to the magnetosheath ion population). Therefore, we may treat them as test particles in externally imposed fields, rather than calculating the fields self-consistently. From the initial source
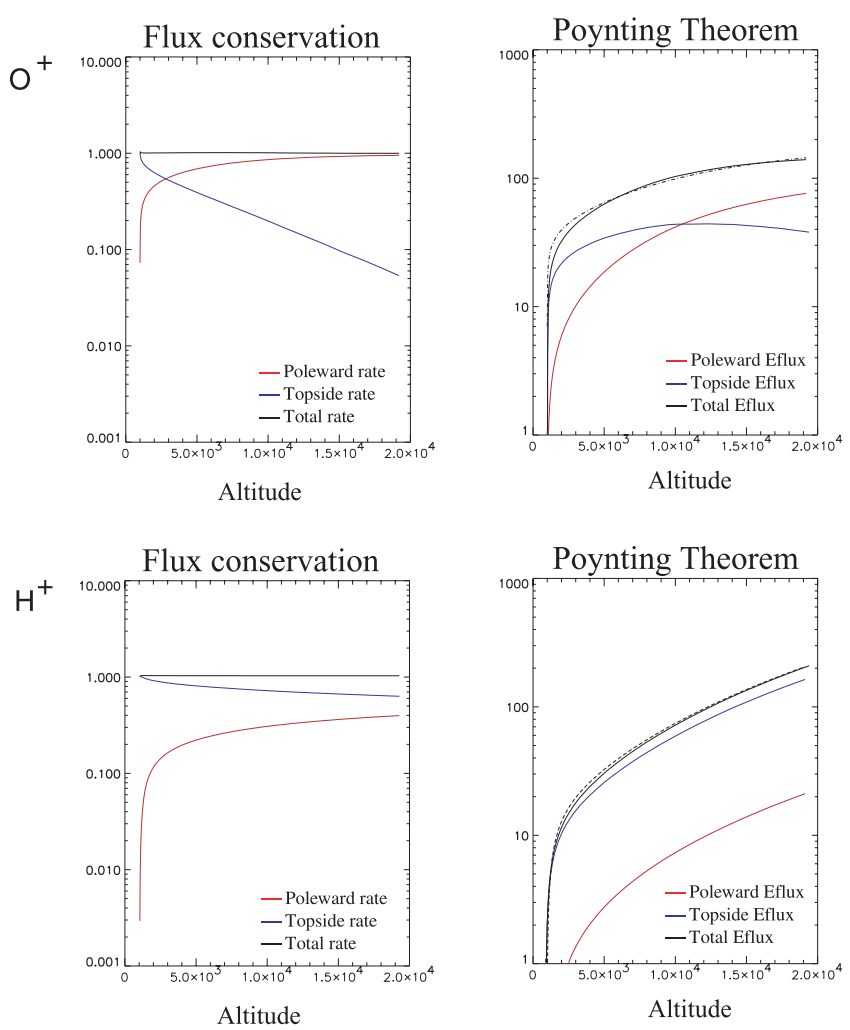

Fig. 5. Evolution of the integrated particle (left-hand side) and the energy density (right-hand side) fluxes of $\mathrm{O}^{+}$(top) and $\mathrm{H}^{+}$(bottom) ions. Red and blue curves correspond to the integrated fluxes over the poleward border $(X=1)$ up to an altitude $z$, and over the horizontal extent of the heating region $(0<X<1)$ at an altitude $z$, respectively. The black curves correspond to the sum of all contributions and the dashed curve (right-hand side) denotes the transferred from the waves to particles below $z$. All quantities are normalized to the values at the low-altitude boundary.

distribution $S_{0}(\boldsymbol{r}, \boldsymbol{V})$ in velocity and space, the calculation of $f_{i}\left(s, X, V_{\|}, V_{\perp}\right)$ proceeds by following the kinetic gyrocenters of test particles. The motion of an ion gyrocenter is described by the set of equations:

$\dot{X}=1 / t_{D}$

$m_{i} \dot{V}_{\|}=q_{i} E_{\|}-m_{i} g(s)-W_{\perp}(\mathrm{d} \ln B / \mathrm{d} s)$,

where $V_{\|}$and $V_{\perp}$ are the parallel and perpendicular velocity components, and $W_{\perp}$ is the perpendicular energy. Equation (5) describes the poleward drift motion along $X$ due to the convection. Equation (6) gives the variation of the parallel velocity under the effect of macroscopic forces, as described in Sect. 2.2.1. In the simulations, we set $E_{\|}=0$ for several reasons. First, introducing $E_{\|} \neq 0$ would be physically meaningful only if its two-dimensional variations can be taken into consideration and in a self-consistent manner that imposes the inclusion of all ion and electron populations in the model (see, for example, Jasperse, 1998). Because we focus on a parametric study associated with a reasonable 
(a)

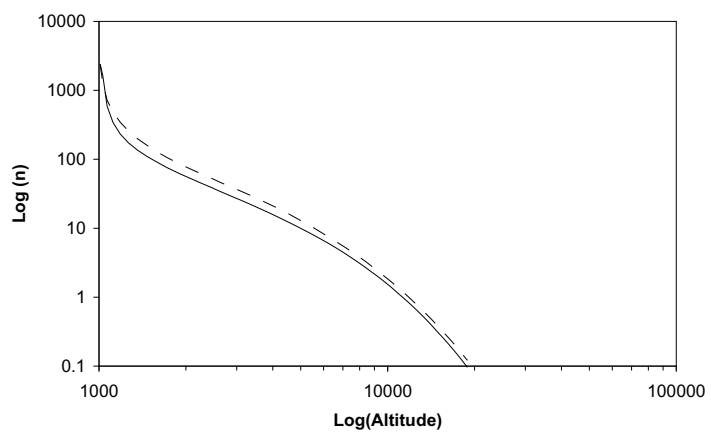

(b)

(c)
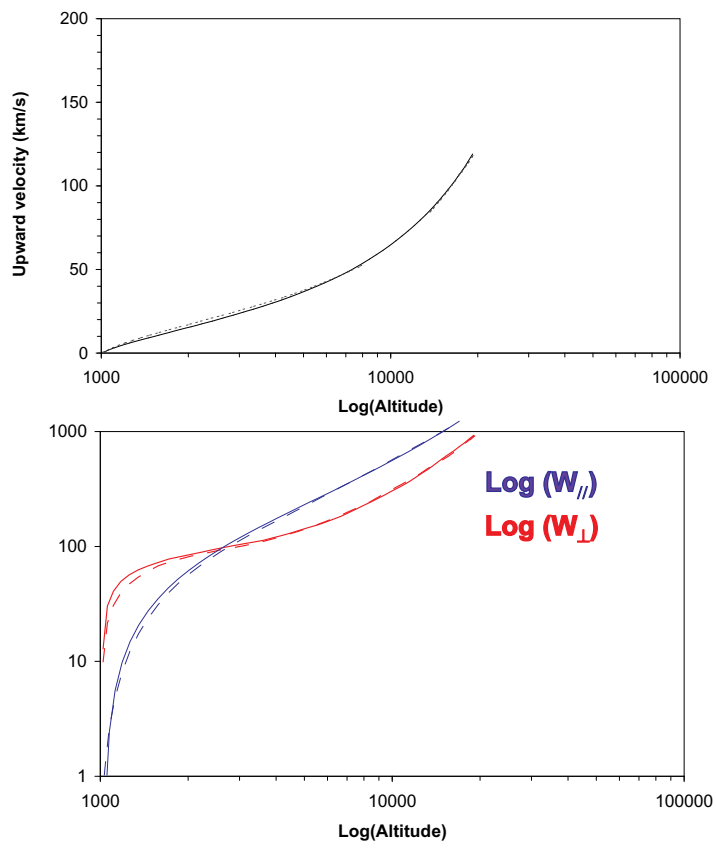

Fig. 6. Evolution of $\mathrm{O}^{+}$moments along the poleward heating boundary: (a) density, (b) upward mean velocity, (c) perpendicular (red) and parallel (blue) mean energies. The solid and dashed curves are associated with a source distribution without temperature and with a parallel temperature of $0.3 \mathrm{eV}$, respectively.

number of free parameters in the simulations, the introduction of an $E_{\|} \neq 0$ component is beyond the scope of the paper. However, it should be noted that in other regions than the dayside cusp/cleft, such as in the downward current premidnight aurora, the field-aligned currents $j_{\|}$and $E_{\|}$are usually stronger. Then $E_{\|}$has a direct consequence in the ion energization, since the heating rate is found to be proportional to the dissipation term $j_{\|} E_{\|}$(Lynch et al., 2002). Hence, in the companion paper, simulations are consequently applied to data sets in the cusp/cleft, where the effect of $E_{\|}$is estimated to be negligible, using the observed electron and ion distributions at high altitude.

In the plane perpendicular to $\boldsymbol{B}$, the effect of a stochastic ion heating results in disturbing the velocity $\boldsymbol{V}_{\perp}$ with a random impulse $\Delta \boldsymbol{V}$ at each time step $\Delta t$. The two components $\Delta V_{x}, \Delta V_{y}$ of $\Delta V_{\perp}$ are generated by a centered Gaussian distribution, such that $\left\langle\Delta V_{x}^{2}\right\rangle=\left\langle\Delta V_{y}^{2}\right\rangle=2 D_{\perp} \Delta t$ (Retterer et al., 1983, 1989). The angle $\theta$ That describes the orientation of $\Delta V_{\perp}$ with respect to the ion gyrophase is assumed to be random. Thus, the variation $\Delta W_{i \perp \text {,res }}$ of the perpendicular energy due to an impulse in a time step $\Delta t$ is given by:

$$
\begin{gathered}
\Delta W_{i \perp, r e s}=\frac{1}{2} m_{i}\left(\boldsymbol{V}_{\perp}+\Delta \boldsymbol{V}_{\perp}\right)^{2}-\frac{1}{2} m_{i}\left|\boldsymbol{V}_{\perp}\right|^{2} \\
=m_{i} V_{\perp} \Delta V_{\perp} \cos \theta+\frac{1}{2} m_{i}\left|\Delta V_{\perp}\right|^{2} .
\end{gathered}
$$

Because $\theta$ is randomly oriented, only the last term of the right-hand side of Eq. (7) contributes statistically to a mean ion heating rate given by:

$\dot{W}_{i \perp}(s)=2 m_{i} D_{i \perp}$.

Inside the heating region $(X<1)$, the time step $\Delta t$ must satisfy the condition $\dot{W}_{i \perp}(s) \times \Delta t \ll W_{i \perp}(s)$, so that the test particle keeps its energy $W_{i}$ and first adiabatic invariant $\mu_{i}$ constant between two random impulses. Typically, we use $\Delta t=\varepsilon W_{i \perp} / \dot{W}_{i \perp}$ with $\varepsilon \sim 0.01$. Outside the heating region, the transverse heating is turned off, and $\Delta t$ is imposed by the adiabatic motion (typically $\left.\Delta t \sim 0.3 / \omega_{c i}\right)$ ).

\subsubsection{Altitude dependence of ion heating}

As discussed in Sect. 1, the wave power spectra exhibit generally a power law in frequency (Gurnett et al., 1984; André et al., 1990): $S(f)=S_{0}\left(f_{0} / f\right)^{\alpha}$, where $S_{0}$ denotes the spectral density at a given frequency $f_{0}$, and $\alpha$ the spectral index. As assumed in previous models (André et al., 1990; Crew et al., 1990), the wave spectrum does not vary versus altitude, and the fraction due to the left-hand polarized component is constant versus frequency. Recently, a statistical profile has been reported for altitudes up to $10000 \mathrm{~km}$ using wave measurements from Akebono correlated with transversely heated ions (Kasahara et al., 2001). Plots on the altitude distribution of the wave power at a frequency of $5 \mathrm{~Hz}$, which corresponds roughly to the $\mathrm{O}^{+}$gyrofrequency at $8000 \mathrm{~km}$, pointed out that the wave power remains approximately constant versus altitude in the dayside cusp/cleft (Plate $5 \mathrm{~b}$ of Kasahara et al., 2001), ranging from about $9 \times 10^{-8}$ to $2.5 \times 10^{-7} \mathrm{~V}^{2} \mathrm{~m}^{-2} \mathrm{~Hz}^{-1}$. This latter result is at least compatible with the assumption that the wave power spectrum is not altitude dependent. Considering these assumptions, since the ion gyrofrequency is proportional to $1 / \mathrm{s}^{3}$, the altitude dependence of the ion heating rate is given by:

$\dot{W}_{i \perp}(s)=\dot{W}_{i \perp}\left(s_{0}\right) \times\left(s / s_{0}\right)^{3 \alpha}$,

where $\dot{W}_{i \perp}\left(s_{0}\right)=\left(q_{i}^{2} / 2 m_{i}\right) S_{L}\left[\left(f_{c i}\left(s_{0}\right)\right)\right]$ denotes the heating rate at the lower boundary distance $s_{0}$. For heavier ions of mass number $M_{i}=m_{i} / m_{H}, \dot{W}_{i \perp}\left(s_{0}\right)$ scales as $M_{i}^{\alpha-1}$. For comparing simulation results for different ions, it is more convenient to associate the altitude profile, given by Eq. (9), with two parameters that do not depend on the type of ion. In doing so, we introduce the second parameter $w_{0}$ given by:

$w_{0}=\left(\frac{m_{H}}{2}\right)^{1 / 3}\left[s_{0} \dot{W}_{i \perp}\left(s_{0}\right) M_{i}^{1-\alpha}\right]^{2 / 3}=$ 
$\left(\frac{m_{H}}{2}\right)^{1 / 3}\left[s_{0} \dot{W}_{H \perp}\left(s_{0}\right)\right]^{2 / 3}$,

where $w_{0}$ has the dimension of energy. On the basis of typical wave observations (see, for example, André et al. (1990), where $0.5<\alpha<2.5$ and $10^{-7}<S\left(f_{c i}\right)<10^{-4} \mathrm{~V}^{2} \mathrm{~m}^{-2} \mathrm{~Hz}^{-1}$ for $\mathrm{O}^{+}$at $s=4 R_{E}, w_{0}$ ranges from 0.1 to $1000 \mathrm{eV}$. Let us note that using a power law dependence versus $s$ is also consistent with the statistical results on the evolution of ion conics along the field lines, which suggest a gradual heating of ions in the cusp/cleft (Peterson et al., 1992; Miyake et al., 1993).

\subsection{Steady-state solution}

There is a fundamental identity between the time integrated solution of an initial value problem and a steady-state problem (Tajima, 1989). Let us note $F(\boldsymbol{r}, \boldsymbol{V}, t)$ for the distribution function associated with test particles at a time $t$ during the simulation. The evolution of $F$ is governed by the dynamical kinetic equation:

$$
\frac{\partial F}{\partial t}+\mathbf{L} F=0
$$

where $\mathbf{L}$ is the kinetic operator, as described in Eq. (3). At $t=0$, the distribution function is determined by the initial condition: $F=S_{0}(r, V)=n_{i}\left(s_{0}\right) \delta\left(s-s_{0}\right) f_{i}(V)$ (injection at $s=s_{0}$ ) and after a time $\Delta T, F=0$ (i.e. no more test particles inside the simulation domain). Integrating Eq. (11) from $t=0$ to $t=\Delta T$, with $f_{i}=\Delta T^{-1} \int_{0}^{\Delta T} F \mathrm{~d} t$, yields:

$\mathbf{L} f_{i}=0$,

with the boundary condition $f_{i}=S_{0}(\boldsymbol{r}, \boldsymbol{V})$ at $s=s_{0}$. Note that the solution $f_{i}\left(s, X, V_{\|}, V_{\perp}\right)$ of this time-averaged equation is the same as in the steady-state kinetic equation for a source $S_{0}$ (Eq. 3). Therefore, we may carry out the solution $f_{i}\left(s, X, V_{\|}, V_{\perp}\right)$ by integrating over time the motion of test particles associated with the source $S_{0}$. In this approach, each test particle contributes to the phase space density of a cell by the time spent in that cell. In the same way, the steady state moments of $f_{i}\left(s, X, V_{\|}, V_{\perp}\right)$ are calculated. As output of a simulation, only the following moments are conserved at any location $(s, X)$ :

$$
\begin{aligned}
& n_{i}(s, X)=\int_{V_{\|}=-\infty}^{V_{\|}=+\infty} \int_{V_{\perp}=0}^{V_{\perp}=+\infty} f_{i}\left(s, X, V_{\|}, V_{\perp}\right) \times \\
& 2 \pi V_{\perp} \mathrm{d} V_{\|} \mathrm{d} V_{\perp} \\
& u_{i}(s, X)=\left(1 / n_{i}\right) \int_{V_{\|}=-\infty}^{V_{\|}=+\infty} \\
& \int_{V_{\perp}=0}^{V_{\perp}=+\infty}\left(V_{\|} f_{i}\left(s, X, V_{\|}, V_{\perp}\right) \times 2 \pi V_{\perp} \mathrm{d} V_{\|} \mathrm{d} V_{\perp}\right. \\
& J_{i}(s, X)=n_{i}(s, X) \times u_{i}(s, X) \times A(s) \\
& w_{i \|}(s, X)=\left(m_{i} / 2 n_{i}\right) \int_{V_{\|}=-\infty}^{V_{\|}=+\infty}
\end{aligned}
$$

$$
\int_{V_{\perp}=0}^{V_{\perp}=+\infty} V_{\|}^{2} f_{i}\left(s, X, V_{\|}, V_{\perp}\right) \times 2 \pi V_{\perp} \mathrm{d} V_{\|} \mathrm{d} V_{\perp}
$$

$$
w_{i \perp}(s, X)=\left(m_{i} / 2 n_{i}\right) \int_{V_{\|}=-\infty}^{V_{\|}=+\infty}
$$$$
\int_{V_{\perp}=0}^{V_{\perp}=+\infty} V_{\perp}^{2} f_{i}\left(s, X, V_{\|}, V_{\perp}\right) \times 2 \pi V_{\perp} \mathrm{d} V_{\|} \mathrm{d} V_{\perp}
$$

$J w_{i}(s, X)=\left(m_{i} / 2\right) \int_{V_{\|}=-\infty}^{V_{\|}=+\infty} \int_{V_{\perp}=0}^{V_{\perp}=+\infty} V_{\|}\left(V_{\|}^{2}+V_{\perp}^{2}\right)$

$f_{i}\left(s, X, V_{\|}, V_{\perp}\right) \times 2 \pi V_{\perp} \mathrm{d} V_{\|} \mathrm{d} V_{\perp}$.

In Eqs. (13)-(18), i denotes the type of ion $\left(\mathrm{H}^{+}\right.$or $\left.\mathrm{O}^{+}\right)$and $n_{i}, u_{i}, J_{i}, w_{i \|}, w_{i \perp}$, and $J w_{i}$ are the ion density, upward drift velocity, ion fluence, mean parallel energy, mean perpendicular energy, and energy density flux, respectively. In Eq. (15), the parameter $A(s)=B\left(s_{0}\right) / B(s)=\left(s / s_{0}\right)^{3}$ is a geometric factor, due to the divergence of the geomagnetic field lines. If the convection drift is turned off, $A(s)$ would correspond to the ratio of cross-section areas of a flux tube between $s_{0}$ and $s$. At $t=0$, about $10^{5} \mathrm{O}^{+}$and $\mathrm{H}^{+}$test particles are injected with an upward mean velocity $u_{i \|}\left(s_{0}\right)$ of $0.4 \mathrm{~km} \mathrm{~s}^{-} 1$ and $5 \mathrm{~km} \mathrm{~s}^{-1}$, respectively. Such values are consistent with typical observations at this altitude (Chandler et al., 1991). The parallel temperature $T_{i \|}\left(s_{0}\right)$ associated with those populations is about a fraction of $1 \mathrm{eV}$. In most of the simulations, we set $T_{i \|}\left(s_{0}\right)=0$. However, we show in Sect. 3 that the results at higher altitudes are insensitive to the moments characteristics of the initial distribution $S_{0}(\boldsymbol{r}, \boldsymbol{V})$.

\section{First results, model tests}

\subsection{Simulation example}

We ran a Monte Carlo simulation with the following parameters: time of drift $t_{D}=500 \mathrm{~s}$, spectral index $\alpha=1$, and $w_{0}=62 \mathrm{eV}$ (see Eq. 11). Figures 2 and 3 show the $\mathrm{O}^{+}$and $\mathrm{H}^{+}$moments, respectively, in a plane $X-s$. Inside the heating region, the density decreases versus $s$ faster than $1 / s^{3}$, in order to satisfy the flux conservation. Conversely, the density decreases as a function of $X$ slowly outside the heating region $(X>1)$. In the panels showing the upward mean velocity $u_{i}$ and the parallel mean energy $w_{i} \|$, we can identify at larger altitudes a dispersion versus $X$ due to the convection. The spread of ion flux tubes also appears clearly in the contours of ion fluence $J_{i}$. This effect is even more significant for heavier ions, such as $\mathrm{O}^{+}$, which have a longer time-of-flight for reaching a geocentric distance $s$. This may also explain the absence of $\mathrm{H}^{+}$at large poleward distances $X$.

Inside the heating region, the perpendicular temperature $T_{\perp}$ and the average pitch angle of ion conics increase gradually versus $s$. This effect is a consequence of the heating profile increasing versus $s$, with the perpendicular energies being progressively transferred into parallel energies via 

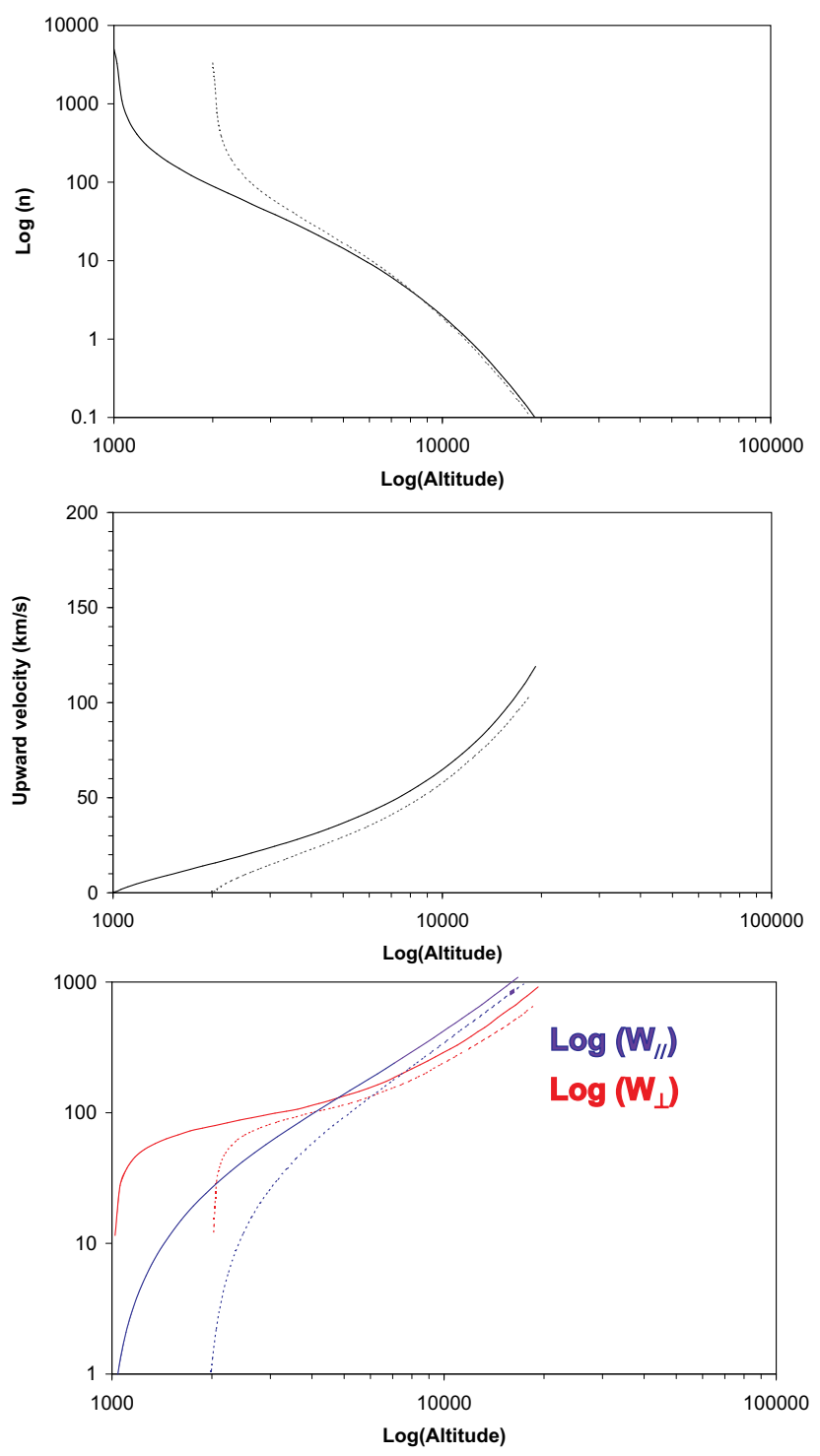

Fig. 7. Evolution of $\mathrm{O}^{+}$moments along the poleward heating boundary: (a) density, (b) upward mean velocity, (c) perpendicular (red) and parallel (blue) mean energies. The solid and dashed curves are associated with a source distribution injected at an altitude of 1000 and $2000 \mathrm{~km}$, respectively.

the mirror force. Poleward of the heating region $(X>1)$, only the mirror force is acting along with gravity for heavy ions, and leads to a steep decrease of $T_{\perp}$ versus $X$. Another counterintuitive result appears in the mean pitch angle panels (Figs. $2 \mathrm{~g}$ and $3 \mathrm{~g}$ ), where $\mathrm{H}^{+}$and $\mathrm{O}^{+}$contours exhibit different values outside the heating region at the lower boundary. Here, we should note that the lower limit for $\mathrm{O}^{+}$is $0^{\circ}$ (downward flow) instead of $90^{\circ}$. Unfortunately, this does not appear in Fig. $2 \mathrm{~g}$, because the color scale is set between $90^{\circ}$ and $180^{\circ}$, in order to keep the discontinuity at the poleward heating boundary $(X=1)$ visible for both ions. We will see in Sect. 4.1.2 that the difference can be explained by the influence of the gravity for low-energy heavy ions, with the corresponding trajectories being parabolic in the low-altitude polar cap (Horwitz, 1984).

\subsection{Tests}

\subsubsection{Validation of ion trajectories}

In the case $t_{D} \rightarrow \infty$ (1-D model, i.e. no convection drift) and neglecting gravity, it is possible to derive an asymptotic solution of the equations describing the motion of a mean particle (Chang et al., 1986). In the limit of high energies, the perpendicular and parallel energy components $\left(W_{\perp}, W_{\|}\right)$ are obtained by using the following expressions:

$$
\begin{aligned}
W_{i \|}(s) & =\frac{9 w_{0} M_{i}^{(2 \alpha-1) / 3}}{2^{1 / 3}[(3 \alpha+1)(6 \alpha+11)]^{2 / 3}}\left(\frac{s}{s_{0}}\right)^{2 \alpha+2 / 3} \\
W_{i \perp}(s) & =\frac{(6 \alpha+2) w_{0} M_{i}^{(2 \alpha-1) / 3}}{2^{1 / 3}[(3 \alpha+1)(6 \alpha+11)]^{2 / 3}}\left(\frac{s}{s_{0}}\right)^{2 \alpha+2 / 3} .
\end{aligned}
$$

Figure 4 shows the evolution of $W_{i \perp}$ and $W_{i \|}$ as functions of $s$ for $w_{0}=62 \mathrm{eV} \mathrm{s}^{-1}$ and $\alpha=1$. The convection drift has been turned off so that the test particle stays inside the heating region. Here, we found that the evolution of $W_{i \perp}$ and $W_{i \|}$ is in good agreement with the mean particle theory developed by Chang et al. (1986). These results allow us to validate the computation of ion trajectories inside the heating region.

\subsubsection{Global conservation laws}

For any simulation we run, it is possible to validate the spatial structure of ion flows by using global conservation laws. By integrating the kinetic equation $\mathbf{L} f_{i}=0$ over all velocity space, we obtain the local conservation equation of particle flux densities:

$\frac{1}{t_{D}} \frac{\partial N_{i}}{\partial X}+\frac{\partial}{\partial s}\left(N_{i} u_{i}\right)=0$,

where $N_{i}=A(\mathrm{~s}) n_{i}(s, X)$. When integrating Eq. (21) over the box limited by $0<X^{\prime}<X$ and $s_{0}<s^{\prime}<s$, we obtain the global conservation law of particle flux densities:

$\int_{s^{\prime}=s_{0}}^{s^{\prime}=s} N_{i}\left(X, s^{\prime}\right) t_{D}^{-1} \mathrm{~d} s^{\prime}+\int_{X^{\prime}=0}^{X^{\prime}=X} N_{i}\left(X^{\prime}, s\right) u_{i}\left(X^{\prime}, s\right) \mathrm{d} X^{\prime}=$

$\int_{s^{\prime}=s_{o}}^{s^{\prime}=s} N_{i}\left(0, s^{\prime}\right) t_{d}^{-1} \mathrm{~d} s^{\prime}+\int_{X^{\prime}=0}^{X^{\prime}=X} N_{i}\left(X^{\prime}, s_{0}\right) u_{i}\left(X^{\prime}, s_{0}\right) \mathrm{d} X^{\prime}$.

By multiplying the kinetic equation by $W=m_{i}\left(V_{\perp}^{2}+V_{\|}^{2}\right) / 2$, and by integrating over all velocity space, we obtain the local conservation equation of energy flux densities:

$$
\begin{aligned}
& \frac{1}{t_{D}} \frac{\partial}{\partial X}\left(N_{i}\left(w_{i}+q_{i} \phi_{i}\right)\right)+ \\
& \frac{\partial}{\partial s}\left(N_{i}\left(Q_{i}+u_{i} q_{i} \phi_{i}\right)\right)=N_{i} \dot{W}_{i \perp}(s),
\end{aligned}
$$

where $q_{i} \phi_{i}$ includes the gravitational and electric potential energy, and $Q_{i}$ denotes the energy flux. Equation (23) may 

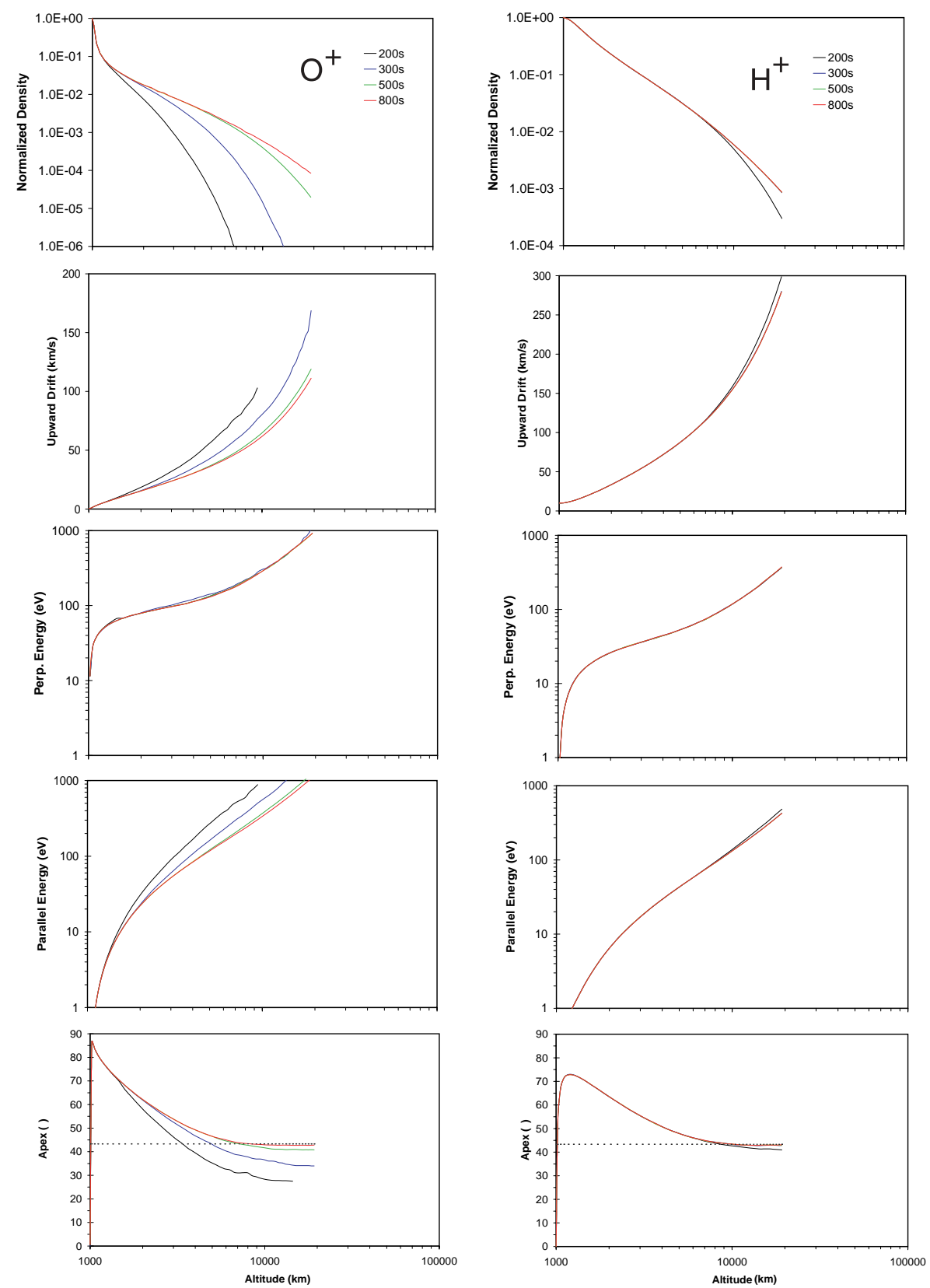

Fig. 8. Evolution of $\mathrm{O}^{+}$(left) and $\mathrm{H}^{+}$(right) moments along the poleward heating boundary. From top to bottom: density, upward mean velocity, perpendicular mean energy, parallel mean energy, and apex. The colours are associated with different values of the residence time as listed in the toper plots.

be simplified by introducing the following approximation: $Q_{i} \approx u_{i} w_{i}$, which physically means that $u_{i}^{2}$ and $w_{i}$ have the same scaling versus $s$ (Crew et al., 1990; Jasperse and Grossbard, 2000). Then, Eq. (23) may be rewritten as:

$\frac{1}{t_{D}} \frac{\partial}{\partial X}\left(N_{i} w_{i}^{*}\right)+\frac{\partial}{\partial S}\left(N_{i} w_{i}^{*} u_{i}\right)=N_{i} \dot{W}_{i \perp}$,

where $w_{i}^{*}=w_{i}+q_{i} \phi_{i}$ is the mean total energy. When integrating Eq. (24) over the box limited by $0<X^{\prime}<X$ and $s_{0}<s^{\prime}<s$, we obtain the global conservation law of energy flux densities for our model:

$\int_{s^{\prime}=s_{0}}^{s^{\prime}=s} N_{i}\left(X, s^{\prime}\right) w_{i}^{*}\left(X, s^{\prime}\right) t_{D}^{-1} \mathrm{~d} S^{\prime}+$

$\int_{X^{\prime}=0}^{X^{\prime}=X} N_{i}\left(X^{\prime}, s\right) w_{i}^{*}\left(X^{\prime}, s\right) u_{i}\left(X^{\prime}, s\right) \mathrm{d} X^{\prime}$

$-\int_{s^{\prime}=s_{0}}^{s^{\prime}=s} n_{i}\left(0, s^{\prime}\right) w_{i}^{*}\left(0, s^{\prime}\right) t_{D}^{-1}-$

$\int_{X^{\prime}=0}^{X^{\prime}=X} N_{i}\left(X^{\prime}, s_{0}\right) w_{i}^{*}\left(X^{\prime}, s_{0}\right) u_{i}\left(X^{\prime}, s_{0}\right) \mathrm{d} X^{\prime}$ 
$=\int_{X^{\prime}=0}^{X^{\prime}=X} \int_{s^{\prime}=s_{0}}^{s^{\prime}=s} N_{i}\left(X^{\prime}, s^{\prime}\right) \dot{W}_{i \perp}\left(X^{\prime}, s^{\prime}\right) \mathrm{d} X^{\prime} \mathrm{d} s^{\prime}$.

This law tells us how much energy density rate goes into (first two terms) and goes out of (next two terms) the box, limited by $0<X^{\prime}<X$ and $s_{0}<s^{\prime}<s$. This is balanced by the transferred power from waves to particles inside the same domain (right-hand side term).

Figure 5 displays, for the previous simulation, the variations of the outflow rate and the energy density rate, as functions of $s$, through the top and the poleward border of a box limited by $0<X<1, s_{0}<s^{\prime}<s$. As shown in Fig. 5a, the sum of the two contributions is still equal to the outflow rate of ions injected at $s=s_{0}$, and therefore Eq. (23) is checked. As shown in Fig. 5b, the conservation of the energy density flux is approximately verified, with the calculation of moments of high order being less accurate numerically.

\subsection{Sensitivity of the results to the boundary conditions}

\subsubsection{Influence of the form of the initial distribution func- tion}

We study here the sensitivity of the characteristics of the initial ion source distribution $S_{0}$ on the steady-state solution of the kinetic equation. Figure 6 shows the profiles of $\mathrm{O}^{+}$moments, along the poleward heating boundary $(X=1)$, with the parameters $w_{0}=62 \mathrm{eV}$ and $\alpha=1$, when introducing a parallel temperature in the source distribution $S_{0}$. The interesting result is the insensitivity of the moment profiles to the source distribution $S_{0}$. Basically, the conjugate action of the diffusion and mirror forces is sufficiently strong so that the form of $S_{0}$ is rapidly rearranged into a heated distribution. For $\mathrm{H}^{+}$ions (not shown), the effect of gravity is negligible and the change on moment profile is therefore insignificant.

\subsubsection{Influence of the low-altitude boundary}

By considering the altitude range $\left(1.5-3 R_{E}\right)$ covered by Interball-2 in the dayside auroral zone, it might be interesting to analyze the sensitivity of the results to the lower geocentric distance boundary, $s_{0}$, of the simulation domain, where ionospheric particles are injected. Figure 7 shows the profiles of $\mathrm{O}^{+}$moments along the poleward heating boundary $(\mathrm{X}=1)$ when considering two different values of $s_{0}$. In both cases, the heating profile is the same as used above. It turns out that the moments are more slightly sensitive to the value of $s_{0}$ than the characteristics of the source distribution $S_{0}$. However, the difference in upward velocity and mean energy components is small, about $\sim 5 \%$ and $\sim 10 \%$, respectively. In addition, there are no changes in the asymptotic evolution of all moment profiles versus $s$.

\subsubsection{Influence of the low-latitude boundary}

Another possible sensitive parameter is the distribution of ions along the equatorward border of the heating region, from which ions flow into the region under the effect of the magnetospheric convection. A few papers reported the presence of transversely accelerated ions at the equatorward edge of the dayside cusp/cleft (André et al., 1988; Whalen et al., 1991). Here, ions may be preheated before entering the energization region, which results in significant density of ions at high altitudes. The contribution of this component to the highaltitude observations usually depends on the residence time $t_{D}$ and the strength of the heating. As mentioned in Sect. 2.1, we should also remind the reader that when considering a homogeneous heating region in latitude and a constant drift, the ions associated with the population flowing from the topside ionosphere and the one from the equatorward boundary do not mix. Here, for the values of $t_{D}$ considered, we found that the equatorward population leads to a more accurate description of the low-latitude side of the heating region and does not drift into the polar cap $(X>1)$. As reported in earlier works using a 2-D model (André et al., 1990), the results at high altitude are not very sensitive to the temperature of the initial distribution. In the companion paper (Bouhram et al., 2003), when applying the results to a few specific data sets, the contribution of the equatorial population is quantified. For doing so, the ion density $n_{i e}$ may be inferred from particle measurements at the satellite altitude $z_{\text {sat }}$. Then, because energization processes are less important equatorward from the heating region, it is reasonable to assume that the density is inversely proportional to the cross-section area $A(s)=\left(s / s_{0}\right)^{3}$ of a magnetic flux tube along the boundary, in order to satisfy the particle conservation law.

The results discussed in Sects. 3.3.1 and 3.3.2 point out that the achieved energies of ionospheric ions are sufficiently important at higher altitudes, so that the characteristics of the ion source distribution have been forgotten, as reported in previous works (Retterer et al., 1987; Crew et al., 1990). In the same way, the low-altitude boundary of the simulation domain has a weak influence on the results at higher altitudes. This justifies that, in our case study, an accurate modelling of physical processes taking place at low altitude $(<2000 \mathrm{~km})$ is not necessary.

\section{Parametric study}

\subsection{Effect of the horizontal drift}

In this section, we quantify the effects of the parameter $t_{D}$, which characterizes the horizontal transport in the poleward $X$ direction, on the spatial structure of ion outflows. Let us remember that when the width $\Delta$ of the heating region is fixed, $t_{D}$ is proportional to the poleward convection drift $v_{p}$. Inside the heating region, we also analyze the differences in terms of ion energization with a 1-D model, where the convection drift is turned off $\left(t_{D} \rightarrow \infty\right)$.

\subsubsection{Influence inside the heating region}

Figure 8 compares $\mathrm{H}^{+}$and $\mathrm{O}^{+}$moment profiles along the poleward heating boundary $(X=1)$ for different values of $t_{D}$ along with the same heating profile $\left(w_{0}=62 \mathrm{eV}, \alpha=1\right)$. Basically, an ion drifts out of the heating region at an altitude 

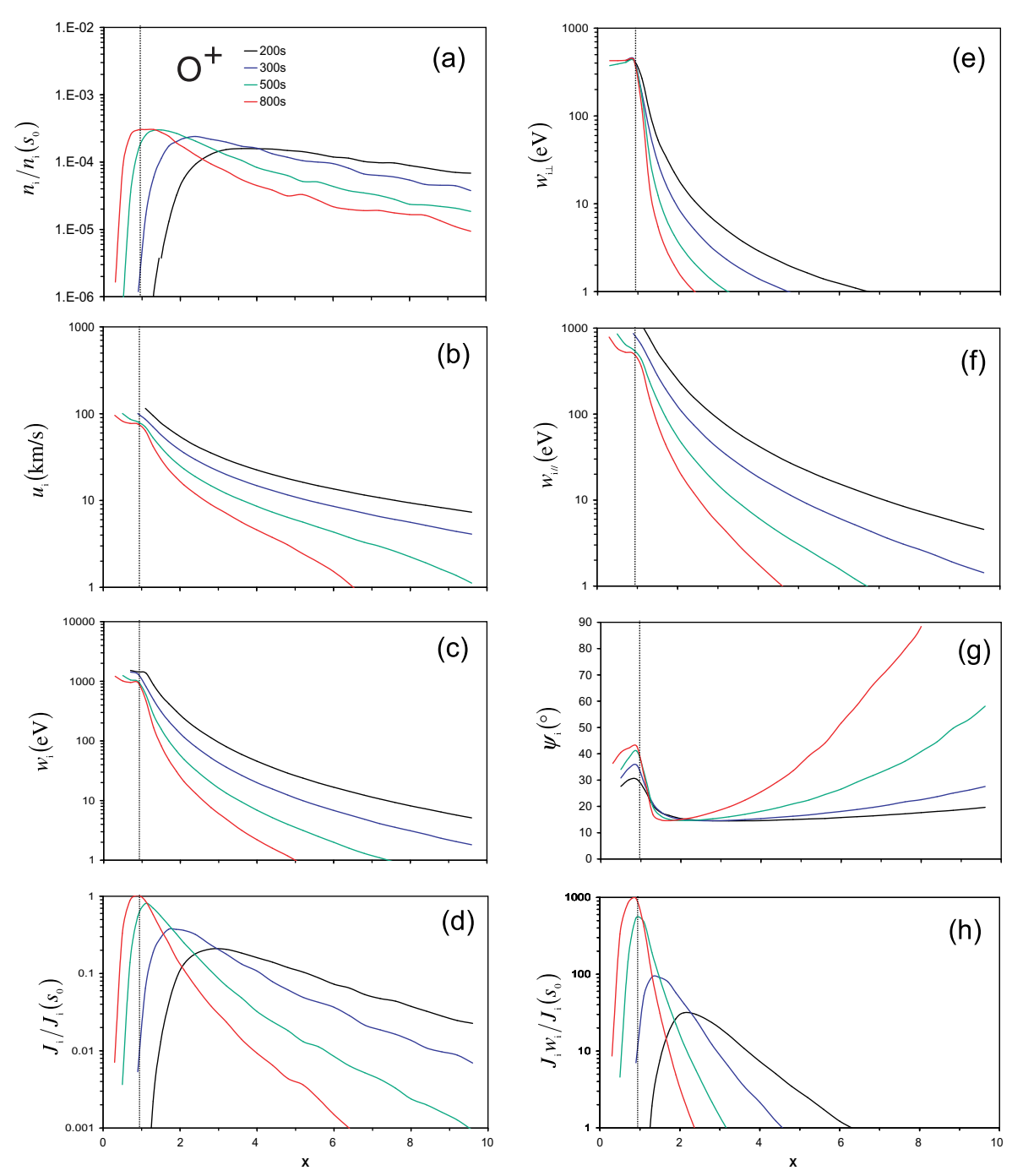

Fig. 9. Evolution of $\mathrm{O}^{+}$moments versus $X$ at an altitude of $2 R_{E}$ : (a) density, (b) upward mean velocity, (c) mean energy, (d) fluence, (e) perpendicular mean energy, (f) parallel mean energy, (g) apex, (h) energy density flux. The moments associated with panels (a), (d) and (h) are normalized with respect to the values at the low-altitude boundary. The colours are associated with different values of the residence time as listed in panel (a).

Table 1. Values of the transferred power per unit area and flux from the source (at $1000 \mathrm{~km}$ ) from waves to $\mathrm{O}^{+}$and $\mathrm{H}^{+}$ions, as a function of $t_{D}$

\begin{tabular}{ccc}
\hline$t_{D}(s)$ & $\left\langle J_{O} w_{O}\right\rangle /\left\langle J_{O}\left(s_{0}\right)\right\rangle(\mathrm{eV})$ & $\left\langle J_{H} w_{H}\right\rangle /\left\langle J_{H}\left(s_{0}\right)\right\rangle(\mathrm{eV})$ \\
\hline 200 & 87 & 160 \\
300 & 139 & 245 \\
500 & 358 & 315 \\
800 & 622 & 354 \\
$+\infty$ & 940 & 375 \\
\hline
\end{tabular}

proportional to its resident time $\left(\leq t_{D}\right)$ inside the heating region. Therefore, density profiles of $n_{i}(s, X=1)$ decrease strongly as $t_{D}$ decreases. In the same way, a more significant fraction of $\mathrm{O}^{+}$ions is spread out of the heating region (in the polar cap) for smaller $t_{D}$. For time-of-flight effects, upward velocity profiles $u_{i}(s, X=1)$ increase strongly as $t_{D}$ decreases: an ion reaching a fixed distance s needs a larger upward velocity as its residence time decreases. Because of such velocity filter effects, ion distribution functions have a smaller parallel temperature than their directed parallel en- 

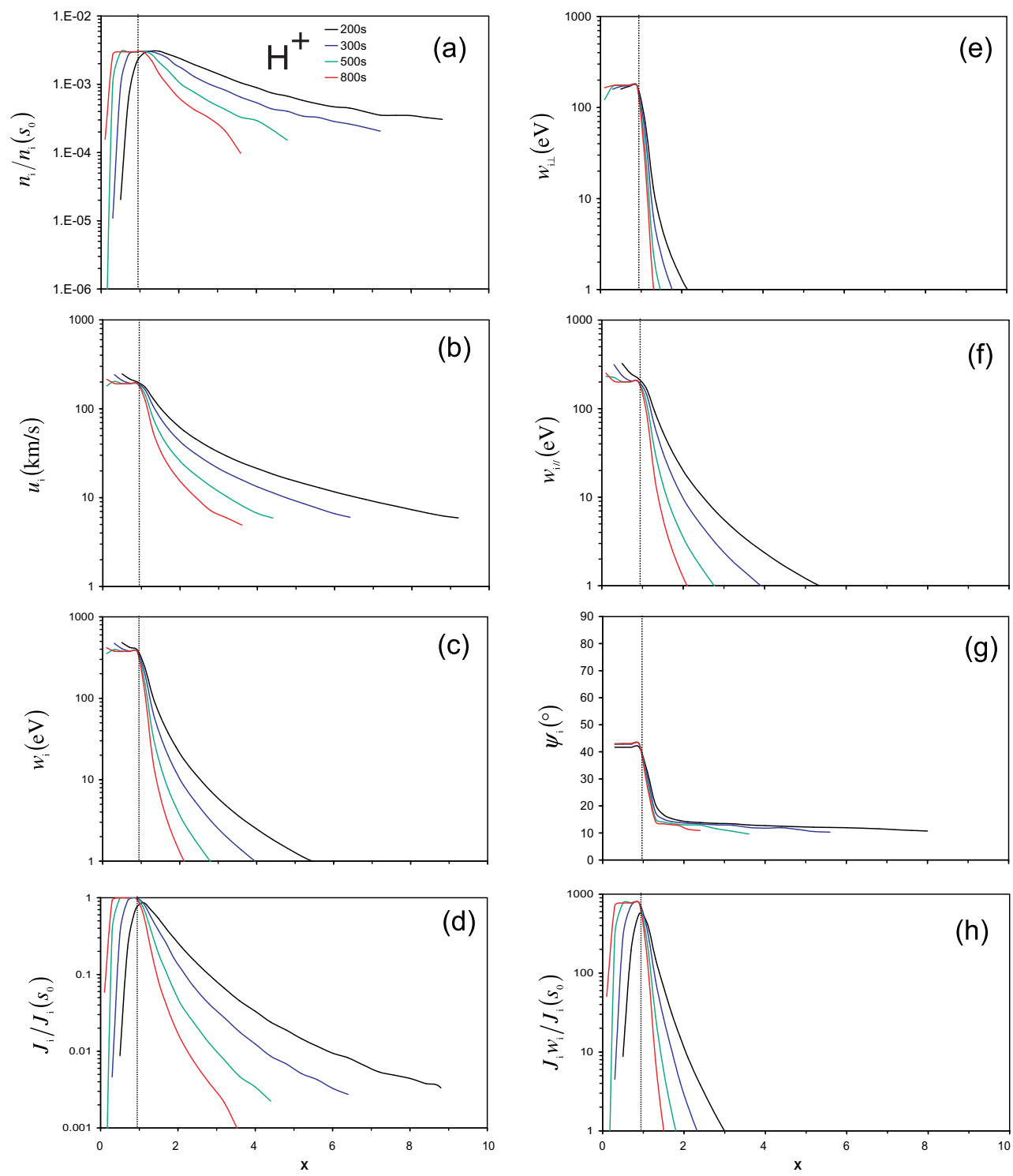

Fig. 10. Evolution of $\mathrm{H}^{+}$moments versus $X$ at an altitude of $2 R_{E}$. The format is the same as in Fig. 8 .

ergy $\left(m_{i} u_{i}^{2} / 2\right)$. Hence, the mean parallel energy $w_{i \|}$ is close to $\left(m_{i} u_{i}^{2} / 2\right)$, and its profile $w_{i \|}(s, X=1)$ increases strongly as $t_{D}$ decreases. On the other hand, perpendicular temperature profiles $w_{i \perp}(s, X=1)$ are insensitive to $t_{D}$. For outflowing ions, the mirror force converts the perpendicular energy into parallel energy along the geomagnetic field lines. Therefore, the achieved perpendicular energies are only due to ICR heating and do not depend on $t_{D}$. The apex angle $\Psi_{i}$, which is a function of the ratio $w_{i \perp} / w_{i \|}$, reaches different asymptotic values. As expected, when $t_{D}$ tends to infinity, $\Psi_{i}$ tends to a value $\left(43.3^{\circ}\right)$ as given by $1-\mathrm{D}$ models (Crew et al., 1990):

$\tan \Psi_{i}=\left(\frac{w_{i \perp}}{w_{i} \|}\right)^{1 / 2}=\frac{(6 \alpha+2)^{1 / 2}}{3}$.

Qualitatively, when $t_{D}$ is larger than the average time of flight for reaching the top of the simulation domain $\left(2 R_{E}\right)$, a sig- nificant part of the ion outflow is still contained inside the heating region. Therefore, all moment profiles have the same shape as given by a 1-D model, on which the ion flux tube is completely contained inside the heating region. $\mathrm{For}^{+}$ions, the parameter $t_{D}$ does not affect the moment profiles. Because the $\mathrm{H}^{+}$mass differs by an important factor $(=16)$ from the $\mathrm{O}^{+}$mass, their average time of flight through the simulation domain remains always smaller than typical values of $t_{D}$

\subsubsection{Influence outside the heating region}

Figures 9 and 10 compare the $\mathrm{O}^{+}$and $\mathrm{H}^{+}$moment profiles versus $X$ at $s_{1}=3 R_{E}$ for the same set of simulation parameters as in Fig. 8. We note that the perpendicular temperature $w_{i \perp}$ and apex angle $\Psi_{i}$ profiles fall down poleward of the heating region $(X>1)$. As previously mentioned, an ion 



Fig. 11. Evolution of $\mathrm{O}^{+}$(left) and $\mathrm{H}^{+}$(right) moments along the poleward heating boundary. From top to bottom: density, upward mean velocity, perpendicular mean energy, and apex. The solid, dashed and dotted curves are associated with different values of the parameter $w_{0}$ as listed in the toper plots.

drifting out of the heating region is only subject to the mirror force and gravity. Because of the absence of transverse energization, heated ion distributions are rapidly folded into upwelling ion distributions inside a thin horizontal region associated with the sharp gradient along $X$ of $w_{i \perp}$ and $\Psi_{i}$.

Poleward of the heating region, ions are filtered owing to their time of flight for reaching $s_{1}=3 R_{E}$, which is inversely proportional to their mean upward drift $u_{i}\left(s_{1}, X\right)$. We will see in the next section that the dispersion $u_{i}\left(s_{1}, X\right)$ is mainly governed by the parameter $t_{D}$. For $X>2$, the apex angle $\Psi_{i}$ of $\mathrm{O}^{+}$increases because of the conjugate action of poleward drift and gravity. When $X$ increases, the field-aligned flow turns downward by increasing in magnitude, and $\Psi_{i}$ tends to $180^{\circ}$. This asymptotic limit is not observed in Fig. 9, because the simulation domain is not sufficiently extended in $X$. ince the effect of gravity is negligible for $\mathrm{H}^{+}, \Psi_{i}$ does not increase for $X>2$.
The effect of the horizontal drift has a major influence on the ionospheric mass loading outside the heating region (polar cap), as evidenced in density $n_{i}\left(s_{1}, X\right)$, fluence $J_{i}\left(s_{1}, X\right)$, and energy density flux $J_{i} w_{i}\left(s_{1}, X\right)$ profiles. By integrating $J_{i}\left(s_{1}, X\right)$ and $J_{i} w_{i}\left(s_{1}, X\right)$ over $X$, we estimate the net outflow rate and the transferred power due to WPI below $s_{1}$ (Eqs. 22 and 25), respectively. For smaller values of $t_{D}$, the energies associated with the maximum fluence decrease strongly for $\mathrm{O}^{+}$. In the same way, the transferred power is as small as $t_{D}$ is small, as given by the area below the curves $J_{i} w_{i}\left(s_{1}, X\right)$. Table 1 gives the transferred power from waves to $\mathrm{O}^{+}$and $\mathrm{H}^{+}$for different values of $t_{D}$. We have added the values as deduced from a 1-D model $\left(t_{D} \rightarrow+\infty\right)$. It turns out that using a 1-D model leads to a strong overestimation of global energy density transfers to heavy ions, such as $\mathrm{O}^{+}$. On the other hand, the effect of the horizontal drift is less significant for $\mathrm{H}^{+}$global energy density transfers. Once again, 

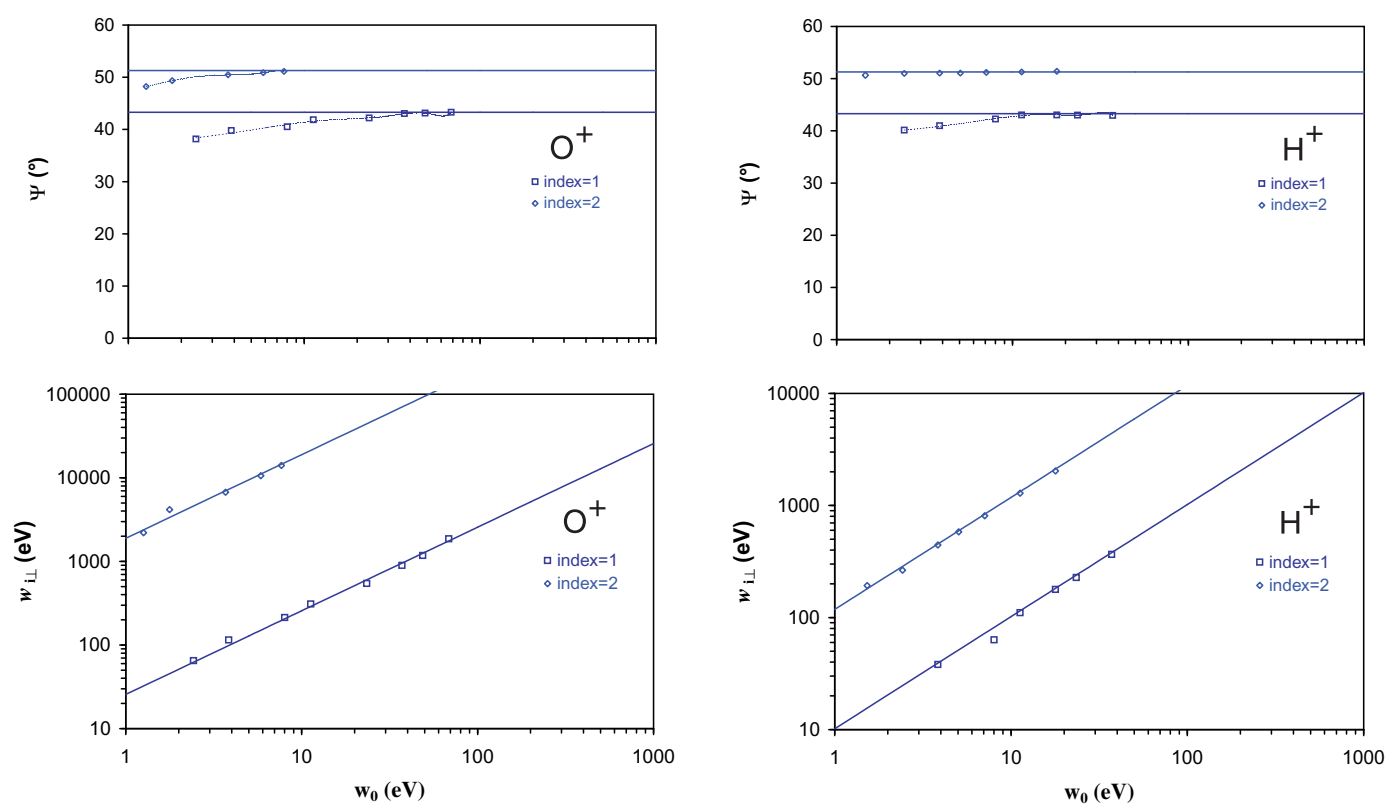

Fig. 12. Evolution of $\mathrm{O}^{+}$(left) and $\mathrm{H}^{+}$(right) apex (top) and mean perpendicular energy (bottom) as functions of $w_{0}$ at the poleward heating boundary and at an altitude of $3 R_{E}$. Squares and diamonds are associated with a spectral index $\alpha=1$ and $\alpha=2$, respectively. The solid curves are associated with the analytic formulas from the 1-D theory.
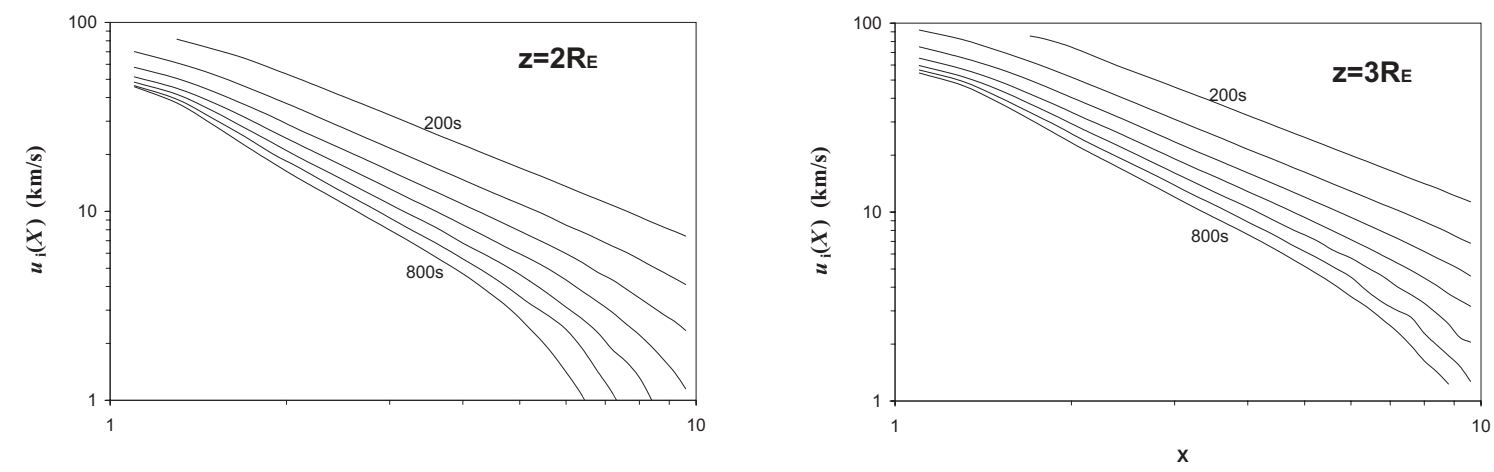

Fig. 13. Evolution of the dispersive patterns of ion upward velocities versus $X$ at two altitudes: $2 R_{E}$ (left) and $3 R_{E}$ (right). The curves are displayed from $t_{D}=200 \mathrm{~s}$ up to $t_{D}=800 \mathrm{~s}$ with a step of $100 \mathrm{~s}$.

this difference is due to their lower mass and leads to average times of flight smaller than $t_{D}$.

\subsection{Influence of the heating profile}

Figure 11 compares the $\mathrm{O}^{+}$and $\mathrm{H}^{+}$moment profiles versus $X$ at $s_{1}=4 R_{E}$ for different heating profiles with $\alpha=1$ fixed, and $t_{D}=500 \mathrm{~s}$. For a fixed value of $t_{D}$, the velocity dispersion in $X$ remains insensitive to the heating parameters. On the other hand, Figs. 9 and 10 have pointed out that the dispersion $u_{i}(s, X)$ exhibits different shapes when considering different values of $t_{D}$. Therefore, we may conclude that the simulated velocity dispersion versus $X$ is a function of $t_{D}$. This result is important when studying an upflowing ion event, because it suggests that the convection drift can be determined from the observed velocity filter effect. Let us introduce the parameters $w_{i \perp p}$ and $\Psi_{i p}$, corresponding to the mean perpendicular energy and the apex, respectively, at the poleward heating boundary (PHB) at a distance $\mathrm{s}$ for a type of ion i. As shown in Fig. 11, the value of $w_{i \perp p}$ increases strongly versus $w_{0}$. Conversely, the value of $\Psi_{i p}$ is approximately constant for $\mathrm{H}^{+}$and slightly dispersed for $\mathrm{O}^{+}\left(\sim 5^{\circ}\right)$. In the following, we will see that $w_{i \perp p}$ and $\Psi_{i p}$ may be used as a probe of the heating parameters $\left(w_{0}, \alpha\right)$.

Figure 12 displays the variation of $\Psi_{i p}$ and $w_{i \perp p}$ at $s=$ $4 R_{E}$ as functions of $w_{0}$ and $\alpha$. Solid curves correspond to the variations of $w_{i \perp p}$ and $\Psi_{i p}$ in the 1-D case. In this approximation, $\Psi_{i p}$ is given by Eq. (26) and depends on $\alpha$ only. For $w_{i \perp p}$, there is no analytic formula. However, on the basis previous studies (Crew et al., 1990; Barghouthi, 1997), $w_{i \perp p}$ may be approximated as the perpendicular energy $W_{i \perp}$ of a single mean ion (Eq. 20). As evidenced in Fig. 12, the values of $w_{i \perp p}$ remain close to the 1-D limit given by Eq. (20). 

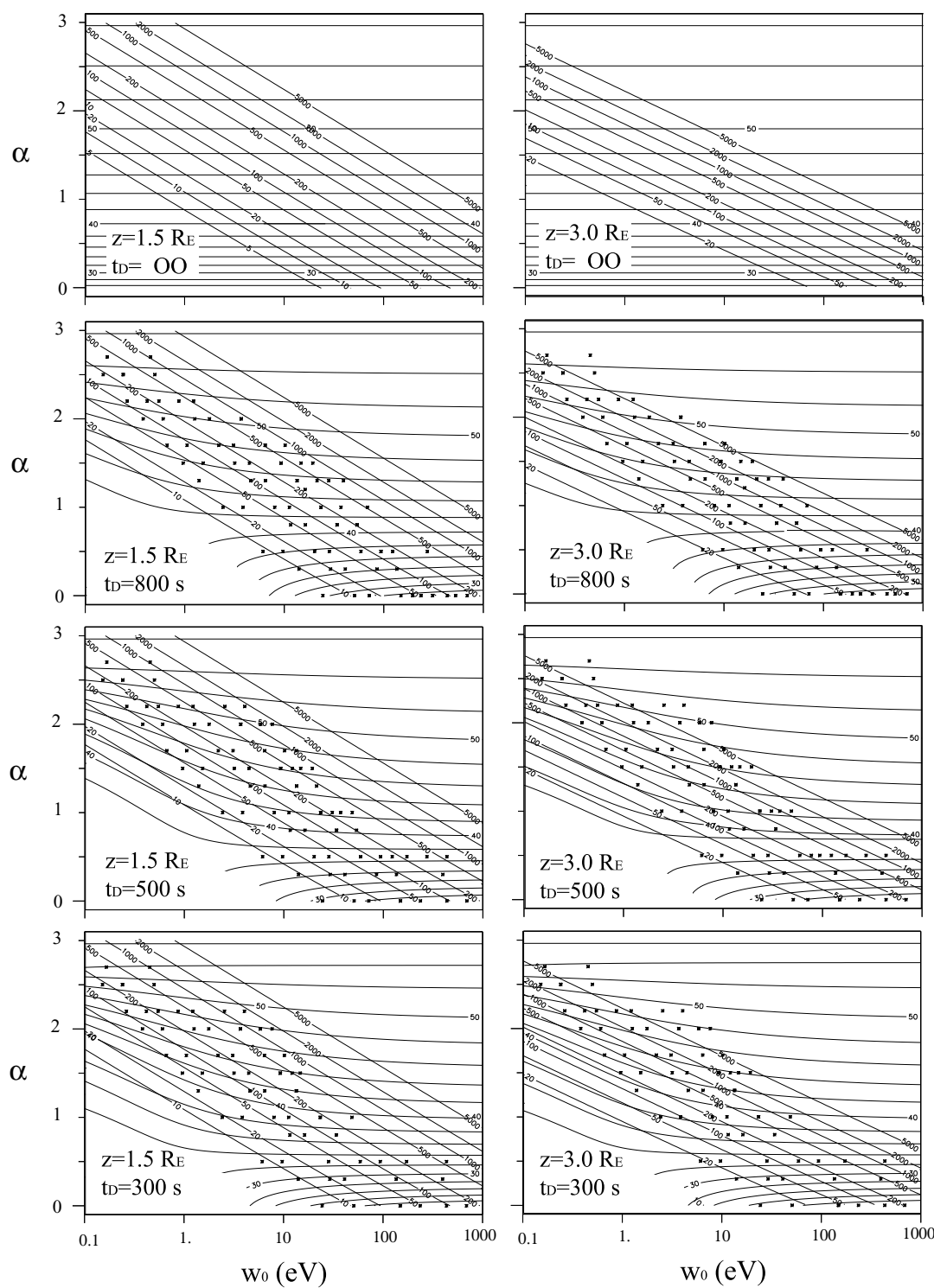

Fig. 14. Patterns in a plane $w_{0}-\alpha$ of the apex and the mean perpendicular energy of $\mathrm{O}^{+}$ions at the poleward edge of the heating region at two altitudes: $1.5 R_{E}$ (left) and $3 R_{E}$ (right). The oblique curves correspond to contours of the mean perpendicular energy (in eV) while the curves approximately horizontal correspond to contours of the apex with a constant step of $2^{\circ}$. Crosses correspond to values inferred from numerical simulations.
Slight differences appear on $\Psi_{i p}$ profiles for $\mathrm{O}^{+}$, where values remain lower than the 1-D limit, because of limited residence times (see Sect. 4.1.1). However, when $\alpha$ increases, $\Psi_{i p}$ changes little and tends to the 1-D limit.

\subsection{Parametric survey}

In the last section, we have studied qualitatively how the simulation parameters $\left(t_{D}, w_{0}, \alpha\right)$ control the moment evolution at higher altitudes. Furthermore, we have established that in the PHB the mean perpendicular energy $w_{i \perp p}$ and the apex $\Psi_{i p}$ associated with high-altitude heated conics are mainly controlled by the heating parameters, $w_{0}$ and $\alpha$. Conversely, outside the heating region, the slope $\mathrm{d} \log \left(u_{i}\right) / \mathrm{d} \log (X)$ characterizing the velocity filter effect is insensitive to the heating parameters and depends on $t_{D}$.

In this section, by gathering the results of a large set of simulation runs, we construct a unique relationship be- tween the triplet $\left[w_{i \perp p}, \Psi_{i p}, \mathrm{~d} \log \left(u_{i}\right) / \mathrm{d} \log (X)\right]$, associated with parameters which are experimentally available, and the triplet $\left(t_{D}, w_{0}, \alpha\right)$ associated with the horizontal drift and the transverse heating.

\subsubsection{Evolution of the velocity filter}

Figure 13 shows the evolution of the ion dispersion $\log \left[u_{i}(X)\right]$ for different values of $t_{D}$ at two geocentric distances. Here, the slope $d \log \left(u_{i}\right) / \operatorname{dLog}(X)$ turns out to be flatter at higher altitudes. Let us consider the flow line $u_{1}$ crossing the point $\left(s_{1}, X_{1}\right)$. From Eq. (21), at a geocentric distance $s_{2}=s_{1}+\Delta s$, the line $u_{1}$ is translated along $X$ by a distance $\Delta X=t_{D}^{-1} \times \Delta s / u_{1}$, inversely proportional to $u_{1}$. Therefore, the slope $\log \left[u_{i}(X)\right]$ decreases flatly versus $X$ between $s_{1}$ and $s_{2}$. 

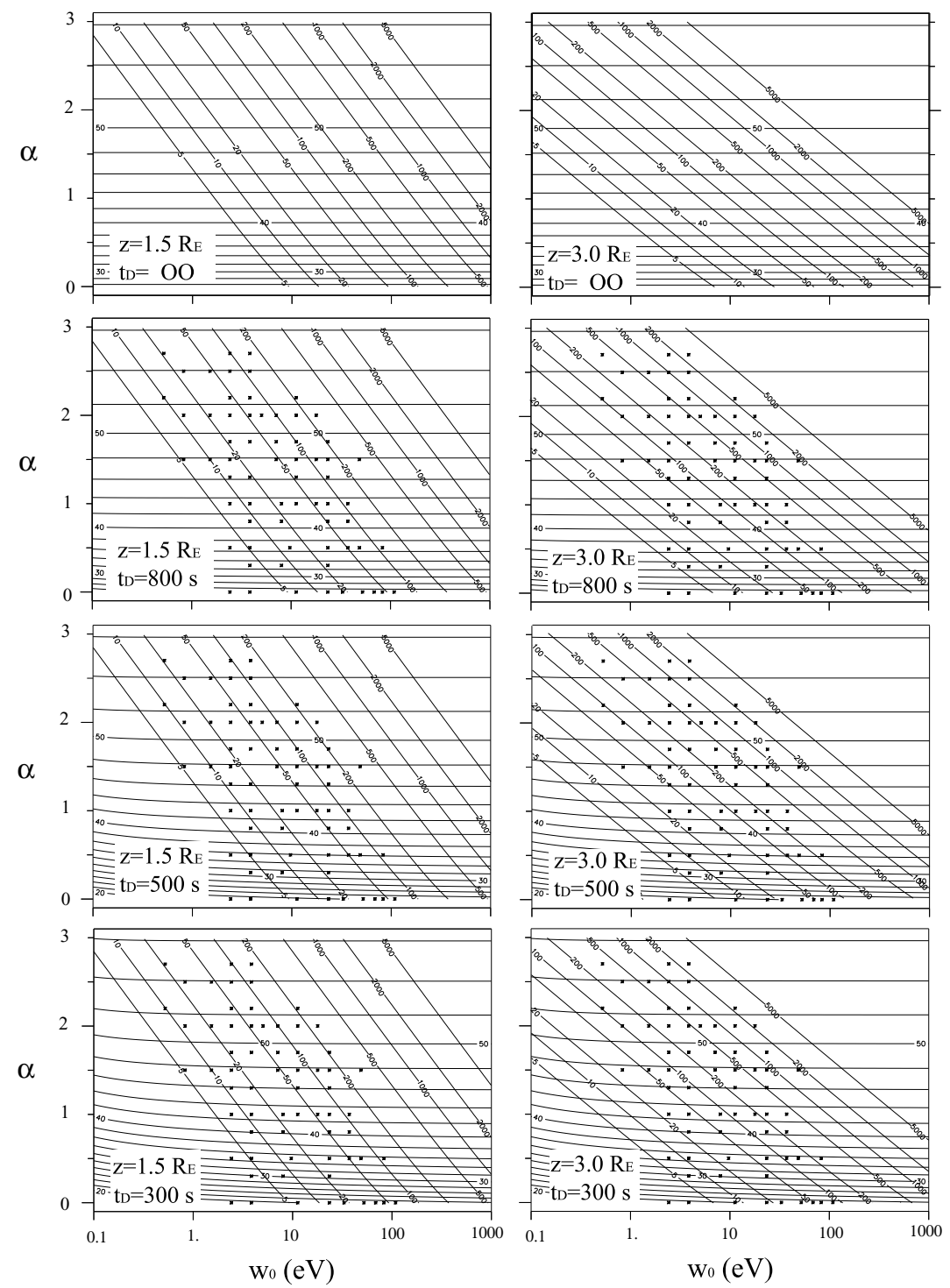

Fig. 15. Patterns in a plane $w_{0}-\alpha$ of the apex and the mean perpendicular energy of $\mathrm{H}^{+}$ions. The format is the same as in Fig. 14.

\subsubsection{Relationship between heating and conic parameters}

From a large set of simulations, we may construct, at a fixed altitude, contour plots of $w_{i \perp p}$ and $\Psi_{i p}$ as functions of the ICR parameters $\left(w_{0}, \alpha\right)$. Figures 14 and 15 provide contour plots of $w_{i \perp p}$ and $\Psi_{i p}$ for $\mathrm{O}^{+}$and $\mathrm{H}^{+}$, respectively, by considering different altitudes and values of $t_{D}$. These contours have been constructed from 1-D fits, similar to Fig. 12. In the limit of higher $w_{0}$ or $\alpha$, the effect of the horizontal drift becomes negligible and the contours follows those given by the 1-D theory (Eqs. 20 and 26). In the domain $\left(w_{0}<1 \mathrm{eV}\right.$, $\alpha<1$ ), contours are not shown because such heating parameters lead to ion distributions associated with insignificant energies $\left(w_{i \perp p}<10 \mathrm{eV}\right)$ for being observed at the PHB. At a fixed altitude, the $w_{i \perp p}$ curves have flatter slope for $\mathrm{O}^{+}$ than $\mathrm{H}^{+}$. By differentiating Eq. (20) and retaining only dom- inant terms, the slope can be expressed as:

$\left[\frac{\partial \alpha}{\partial \log \left(w_{0}\right)}\right]_{w_{i} \perp} \approx \frac{1}{\log \left[M_{i}^{2 / 3}\left(s / s_{0}\right)^{2}\right]}$.

Therefore, the slope of $w_{i \perp p}$ contour decreases with respect to the ion mass and the altitude. Furthermore, there is a single intersection between a $w_{i \perp p}$ contour and a $\Psi_{i p}$ contour. Therefore, a couple $\left(w_{0}, \alpha\right)$ leads at a fixed altitude to a unique ion conic form at the PHB and is characterized by the couple of moments $\left(w_{i \perp p}, \Psi_{i p}\right)$.

\section{Summary and conclusion}

In this paper, we have studied the spatial structure, from $1000 \mathrm{~km}$ up to $20000 \mathrm{~km}$ in altitude, of dayside ionospheric ion outflow as a function of ion heating parameters and the convection drift using a two-dimensional (2-D), Monte Carlo, trajectory-based code. Since no analytic theory is 
available in 2-D, the results of the model have been tested successfully on the basis of global conservation laws.

In contrast to previous work based on 1-D simulation, the introduction of a poleward convection drift leads to a limited residence time $t_{D}$ of ions when being heated in the dayside cusp/cleft. Residence time effects turn out to be important by modifying the density patterns of $\mathrm{O}^{+}$outflows, while residence time effects on density patterns are small for $\mathrm{H}^{+}$ outflows. These effects also control the dispersive energy patterns of outflows poleward of the heating region (i.e. in the polar cap). Conversely, inside the heating region, limited residence times lead to moderate and weak effects in the angle and energy characteristics associated with $\mathrm{O}^{+}$and $\mathrm{H}^{+}$ conics, respectively. Such parameters are to the first order controlled by the strength of ion transverse heating, as characterized in the model by the couple of parameters $\left(w_{0}, \alpha\right)$. In terms of energy density flux exchanges, residence time effects are important for heavy populations, such as $\mathrm{O}^{+}$. Because the ion density is overestimated using a 1-D model, the introduction of a limited residence time $t_{D}$ leads to smaller transfers of power between the waves and the $\mathrm{O}^{+}$ions inside the heating region.

The main conclusion of the paper is that the transport patterns of ion outflows when associated with a reasonable number of free parameters $\left(t_{D}, w_{0}, \alpha\right)$, lead to unique features in space. In a companion paper (Bouhram et al., 2003), providing that our assumptions are satisfied, we reverse the process by determining the strength of ion heating $\left(w_{0}, \alpha\right)$ for a large number of data sets in the dayside cusp/cleft from the Interball-2 satellite.

Acknowledgements. The Interball Project was accomplished in the frame of contract N025-7535/94 with the Russian Space Agency (RKA). The Hyperboloid experiment was financially supported by CNES under the auspices of grants covering the period 1985 to 2002.

Topical Editor T. Pulkkinen thanks E. Lund and another referee for their help in evaluating this paper.

\section{References}

André, M., Matson, L., Koskinen, H., and Erlandson, R.: Local transverse ion energization in and near the polar cusp, Geophy. Res. Lett., 15, 107, 1988.

André, M., Crew, G. B., Peterson, W. K., Persoon, A. M., Pollock, C. J., and Engebretson, M. J.: Ion heating by broadband lowfrequency waves in the cusp/cleft, J. Geophy. Res., 95, 20809 , 1990.

André, M. and Yau, A. W.: Theories and observations of ion energization and outflow in the high latitude magnetosphere, Space Sci. Rev., 80, 27-48, 1997.

Barghouthi, I. A.: Effects of wave-particle interactions on $\mathrm{H}^{+}$and $\mathrm{O}^{+}$outflow at high latitude: A comparative study, J. Geophys. Res., 102, 22 065-22 075, 1997.

Bouhram, M., Malingre, M., Jasperse, J. R., Dubouloz, N., and Sauvaud, J.-A.: Modeling transverse heating and outflow of ionospheric ions from the dayside cusp/cleft: 2 Applications, Ann. Geophysicae, this issue, 2003.
Chandler, M. O., Moore, T. E., and Waite, Jr., J. H.: Observations of polar ion outflows, J. Geophys. Res., 96, 1421, 1991.

Chang, T., Crew, G. B., Hershkowitz, N., Jasperse, J. R., Retterer, J. M., and Winningham, J. D.: Transverse acceleration of oxygen ions by electromagnetic ion cyclotron resonance with broadband left-hand-polarized waves, Geophys. Res. Lett., 13, 636639, 1986.

Chen, S.-H., Boardsen, S. A., Fung, S. F., Green, J. L., Kessel, R. L., Tan, L. C., Eastman, T. E., and Craven, J. D.: Exterior and interior polar cusps: Observations from Hawkeye, J. Geophys. Res., 102, 11335-11 348, 1997.

Cladis, J. B.: Parallel acceleration and transport of ions from polar ionosphere to plasma sheet, Geophys. Res. Lett., 13, 893-896, 1986.

Crew, G. B. and Chang, T.: Asymptotic theory of ion conic distributions, Phys. fluids, 28, 2382-2394, 1985.

Crew, G. B., Chang, T., Retterer, J. M., Peterson, W. K., Gurnett, D. A., and Huff, R. L.: Ion cyclotron resonance heated conics: theory and observations, J. Geophys. Res., 95, 3959, 1990.

Dubouloz, N., Delcourt, D., Malingre, M., Berthelier, J.-J., and Chugunin, D.: Remote analysis of cleft ion acceleration using thermal plasma measurements from Interball Auroral Probe, Geophys. Res. Lett., 25, 2925, 1998.

Dubouloz, N., Bouhram, M., Senior, C., Delcourt, D., Malingre, M., and Sauvaud, J.-A.: Spatial structure of the cusp/cleft ion fountain: A case study using a magnetic conjugacy between Interball AP and a pair of SuperDARN radars, J. Geophys. Res., 106, 261-274, 2001.

Gurnett, D. A., Huff, R. L., Menietti, J. D., Burch, J. L., Winningham, J. D., and Shawhan, S. D.: Correlated low-frequency electric and magnetic noise along the auroral field lines, J. Geophys. Res., 89, 8971-8985, 1984.

Horwitz, J. L.: Features of ion trajectories in the polar magnetosphere, Geophys. Res. Lett., 11, 1111, 1984.

Horwitz, J. L. and Lockwood, M.: The Cleft ion fountain, a twodimensional kinetic model, J. Geophys. Res., 90, 9749, 1985.

Horwitz, J. L.: Velocity filter mechanism for ion bowl distributions (Bimodal conics), J. Geophys. Res., 91, 4513, 1986.

Ichimaru, S.: Basic principles of plasma physics: A statistical approach, W. A. Benjamin, Reading, Mass., 1973.

Jasperse, J. R.: Ion heating, electron acceleration and the selfconsistent parallel E-field in downward auroral current regions, Geophys. Res. Lett., 25, 3485-3488, 1998.

Jasperse, J. R. and Grossbard, N.: The Alfvén-Falthammar formula for the parallel E-field and its analogue in downward auroralcurrent regions, IEEE Trans. Plasma Sci., 28, 1874, 2000.

Kasahara, Y., Hosoda, T., Mukai, T., Watanabe, S., Kimura, I., Kojima, H., and Niitsu, R.: ELF/VLF waves correlated with transversely accelerated ions in the auroral region observed by Akebono, J. Geophys. Res., 106, 21 123-21 136, 2001.

Kintner, P. M.: Observations of velocity shear driven plasma turbulence, J. Geophys. Res., 81, 5114-5122, 1976.

Knudsen, D. J., Whalen, B. A., Abe, T., and Yau, A.: Temporal evolution and spatial dispersion of ion conics: evidence of a polar cusp heating wall, in: Solar System Plasmas in Space and Time, Geophys. Monogr. Ser., vol. 84, (Eds.) Burch, J. L. and Waite, Jr., J. H., AGU, Washington D.C., 163-169, 1994.

Lockwood, M., Waite, Jr., J. H., Moore, T. E., Johnson, J. F. E., and Chappell, C. R.: A new source of suprathermal $\mathrm{O}^{+}$ions near the dayside polar cap boundary, J. Geophys. Res., 90, 4099, 1985a.

Lockwood, M., Chandler, M. O., Horwitz, J. L., Waite, Jr., J. H., Moore, T. E., and Chappell, C. R.: The cleft ion fountain, J. 
Geophys. Res., 90, 9736, 1985b.

Lund, E. J., Moebius, E., Carlson, C. W., Ergun, R. E., Kistler, L. M., Klecker, B., Klumpar, D. M., McFadden, J. P., Popecki, M. A., Strangeway, R. J., and Tung, Y. K.: Transverse ion acceleration mechanism in the aurora at solar minimum: occurrence distributions, J. Atmos. Terr. Phys., 62, 467-475, 2000.

Lynch, K. A., Bonnell, J. W., Carlson, C. W., and Peria, W. J.: Return current region aurora: $\mathrm{E}_{\|}, \mathrm{j}_{\mathrm{z}}$, particle energization, and broadband ELF wave activity, J. Geophys. Res., 107(A7), 10.1029/2001JA900134, 2002.

Miyake, W., Mukai, T., and Kaya, N.: On the evolution of ion conics along the field line from EXOS-D observations, J. Geophys. Res., 98, 11 127, 1993.

Moore, T. E., Lundin, R., Alcaydé, D., André, M., Ganguli, S. B., Temerin, M., and Yau, A.: Source processes in the high-altitude ionosphere, Space Sci. Rev., 88, 7-84, 1999.

Newell, P. T. and Meng, C.-I.: Mapping the dayside ionosphere to the magnetosphere according to particle precipitation characteristics, Geophys. Res. Lett., 19, 609-612, 1992.

Norqvist, P., André, M., Eliasson, L., Eriksson, A. I., Blomberg, L., Lühr, H., and Clemmons, J. H.: Ion cyclotron heating in the dayside magnetosphere, J. Geophys. Res., 101, 13 179, 1996.

Norqvist, P., André, M., and Tyrland, M.: A statistical study of ion energization mechanisms in the auroral region, J. Geophys. Res., 103, 23 459-23 473, 1998.

Øieroset, M., Yamauchi, M., Liszka, L., and Christon, S. P.: Energetic ion outflow from the dayside ionosphere and its relationship to the interplanetary magnetic field and substorm activity, J. Atmos. Terr. Phys., 62, 485-493, 2000.
Peterson, W. K., Collin, H. L., Doherty, M. F., and Bjorklund, C. $\mathrm{M} .: \mathrm{O}^{+}$and $\mathrm{He}^{+}$restricted and extended (bi-modal) ion conic distributions, Geophys. Res. Lett., 19, 1439-1442, 1992.

Retterer, J. M., Chang, T., and Jasperse, J. R.: Ion acceleration in the suprauroral region: a Monte Carlo model, Geophys. Res. Lett., 10, 583-586, 1983.

Retterer, J. M., Chang, T., Crew, G. B., Jasperse, J. R., and Winningham, J. D.: Monte Carlo modeling of ionospheric oxygen acceleration by cyclotron resonance with broad-band electromagnetic turbulence, Phys. Rev. Lett., 59, 148-151, 1987.

Retterer, J. M., Chang, T., and Jasperse, J. R.: Particle acceleration by intense auroral VLF turbulence, in: Physics of Space Plasmas, (Eds) Chang, T., Crew, G. B., and Jasperse, J. R., Sci. Publishers, Cambridge, Angleterre, 119, 1989.

Ruohoniemi, J. M. and Greenwald, R. A.: Statistical patterns of high-latitude convection obtained from Goose Bay HF radar observations, J. Geophys. Res., 101, 21 473, 1996.

Shelley, E. G., Johnson, R. G., and Sharp, R. D.: Satellite observations of energetic heavy ions during a geomagnetic storm, $\mathrm{J}$. Geophys. Res., 77, 6104, 1972.

Tajima, T.: Computational Plasma Physics-with Applications to Fusion and Astrophysics, Addison-Wesley (Benjamin Frontier Series, Reading, MA), 1989.

Thelin, B., Aparicio, B., and Lundin, R.: Observations of upflowing ionospheric ions in the mid-altitude cusp/cleft region with the Viking satellite, J. Geophys. Res., 95, 5931-5939, 1990.

Whalen, B. A., Watanabe, S., and Yau, A. W.: Observations in the transverse energization region, Geophys. Res. Lett., 18, 725, 1991. 